\title{
HYPERBOLIC AND DIOPHANTINE ANALYSIS
}

\author{
BY SERGE LANG
}

Dedicated to F. Hirzebruch

In this survey we consider Kobayashi hyperbolicity, in which there is an interplay between five notions:

analytic notions of distance and measure;

complex analytic notions;

differential geometric notions of curvature (Chern and Ricci form);

algebraic notions of "general type" (pseudo ampleness);

arithmetic notions of rational points (existence of sections).

I am especially interested in the relations of the first four notions with diophantine geometry, which historically has intermingled with complex differential geometry. One of the main points of this survey is to arrive at a certain number of conjectures in an attempt to describe at least some of these relations coherently.

Throughout this article, by an algebraic set we mean the set of zeros of a finite number of homogeneous polynomials

$$
P_{j}\left(x_{0}, \ldots, x_{N}\right)=0, \quad j=1, \ldots, m
$$

in projective space $\mathbf{P}^{N}$ over $\mathbf{C}$, so $x_{0}, \ldots, x_{N}$ are the projective coordinates. An algebraic set will be called a variety if it is irreducible - that is, the polynomials can be chosen so that they generate a prime ideal. If $X$ has no singularities, then $X$ is also a complex manifold. In any case, $X$ has a complex structure. From now on, we let $X$ denote a variety.

Among the possible complex analytic properties of $X$ we shall emphasize that of being hyperbolic. There are several equivalent definitions of this notion, and one of them, due to Brody, is that every holomorphic map of $\mathbf{C}$ into $X$ is constant. At the beginning of this article, we shall give three possible characterizations, including Kobayashi's original definition, and prove the equivalence between them, following Brody.

On the other hand, $X$ also has an algebraic structure. For one thing, taking the algebraic subsets of $X$ as closed subsets defines the Zariski topology. Thus the Zariski closure of a set $S$ is the smallest algebraic set containing $S$, and is equal to the intersection of all algebraic sets containing $S$. Furthermore, the polynomials $P_{j}$ have coefficients in some field $F_{0}$, finitely generated over the rational numbers, and this gives rise to diophantine properties as follows.

Received by the editors March 13, 1985 and, in revised form, July 3, 1985.

1980 Mathematics Subject Classification (1985 Revision). Primary 14J99, 14G95, 32H20, 11 D41. 
Let $F$ be a field containing $F_{0}$. A point with projective coordinates $\left(x_{0}, \ldots, x_{n}\right)$ is said to be rational over $F$ if the affine coordinates

$$
x_{0} / x_{j}, \ldots, x_{n} / x_{j}
$$

lie in $F$ for some index $j$ such that $x_{j} \neq 0$, and hence for every such index. The set of $F$-rational points of $X$ is denoted by $X(F)$. When we speak of rational points, we shall always mean rational points in some field of finite type over the rationals, that is, finitely generated over the rationals. No other qualification will be made.

We shall say that $X$ (or a Zariski open subset) is mordellic if it has only a finite number of rational points in every finitely generated field over $\mathbf{Q}$ according to our convention. I once conjectured that a hyperbolic variety is mordellic. We shall put this conjecture in a much broader context.

Let $X$ be any variety. We define the analytic exceptional set $\operatorname{Exc}(X)$ of $X$ to be the Zariski closure of the union of all images of non-constant holomorphic maps $f: \mathbf{C} \rightarrow X$. Thus $X$ is hyperbolic if and only if this exceptional set is empty. In general the exceptional set may be the whole variety, $X$ itself. I conjecture that $X-\operatorname{Exc}(X)$ is mordellic.

It is also a problem to give an algebraic description of the exceptional set, giving rise to the converse problem of showing that the exceptional set is not mordellic, and in fact always has infinitely many rational points in a finite extension of a field of definition for $X$. We define the algebraic exceptional set $\operatorname{Exc}_{\text {alg }}(X)$ to be the union of all non-constant rational images of $\mathbf{P}^{1}$ and abelian varieties in $X$. (An abelian variety is a variety which is complex analytically isomorphic to a complex torus.) I would conjecture that the analytic exceptional set is equal to the algebraic one. A subsidiary conjecture is therefore that $\operatorname{Exc}_{\mathrm{alg}}(X)$ is closed, and that $X$ is hyperbolic if and only if every rational map of $\mathbf{P}^{1}$ or an abelian variety into $X$ is constant. Similarly, until the equality between the two exceptional sets is proved, one has the corresponding conjecture that the complement of the algebraic exceptional set is mordellic.

Observe that the equality

$$
\operatorname{Exc}(X)=\operatorname{Exc}_{\mathrm{alg}}(X)
$$

would give an algebraic characterization of hyperbolicity. Such a characterization implies for instance that if a variety is defined over a field $F_{0}$ as above, and is hyperbolic in one imbedding of $F_{0}$ in $\mathbf{C}$, then it is hyperbolic in every imbedding of $F_{0}$ in $\mathbf{C}$, something which is by no means obvious, given the analytic definitions of "hyperbolic" in $\$ \S 1$ and 2.

We wish to characterize those varieties such that the exceptional set is a proper subset. We shall give conjecturally a number of equivalent conditions, which lead us into complex differential geometry, and algebraic geometry as well as measure theory.

The properties having to do with hyperbolicity from the point of view of differential geometry have been studied especially by Grauert-Reckziegel, Green, Griffiths, and Kobayashi. Such properties have to do with "curvature". In $\$ 4$ we reproduce the proof that under certain "curvature" conditions, the variety is hyperbolic. Also in $\$ 4$ we consider a weakening of this notion, 
namely measure hyperbolic, due to Kobayashi. The difference lies in looking at positive $(1,1)$-forms or volume $(n, n)$-forms on a variety of dimension $n$. One key aspect is that of the Ahlfors-Schwarz lemma, which states that a holomorphic map is measure decreasing under certain conditions.

In $\$ 3$ we describe how to associate a $(1,1)$-form, the $\operatorname{Ricci}$ form $\operatorname{Ric}(\Psi)$, to a volume form $\Psi$. The positivity of the Ricci form is related in a fundamental way to hyperbolicity. In the differential geometric part of this article, the Schwarz lemma and the formalism attached to the positivity of the Chern form

$$
d d^{c} \log |\sigma|^{2}
$$

where $|\sigma|$ is a length function of some sort, provide a uniform thread weaving through all the questions.

We shall also be led to weaken certain properties. Roughly speaking, given a property, the weakening of this property obtained by requiring that it holds only outside a proper algebraic subset may be called its pseudofication. Thus we shall deal with pseudo volume forms, for which we allow zeros on a proper analytic subset. The precise definition is given in $\S 4$. We can also say that $X$ is pseudo hyperbolic if the exceptional set is a proper subset. We say that $X$ is pseudo mordellic if there exists a proper algebraic subset $Y$ such that $X-Y$ is mordellic.

In the direction of algebraic geometry, let $T=T_{X}$ be the complex tangent bundle, and $T^{\vee}$ the cotangent bundle dual of $T$, assuming now that $X$ is non-singular. Let

$$
K=K_{X}=\bigwedge^{\text {top }} T^{\vee}
$$

be the canonical bundle of differential forms of top degree. We say that $X$ is canonical if there exists a positive integer $m$ such that a basis $\left(s_{0}, \ldots, s_{N}\right)$ for the space of sections $H^{0}\left(X, K^{\otimes m}\right)$ gives an imbedding

$$
x \mapsto\left(s_{0}(x), \ldots, s_{N}(x)\right)
$$

of $X$ into $\mathbf{P}^{N}$. We say that $X$ is pseudo canonical (or of general type) if there exists some $m$ such that the map is defined outside a proper algebraic subset, and gives a projective imbedding of the complement. A singular variety is called pseudo canonical if a desingularization is pseudo canonical.

Then the problem is to determine whether the following conditions are equivalent:

P1. $X$ is pseudo canonical.

P2. $X$ is pseudo mordellic.

P3. $X$ is pseudo hyperbolic.

$\mathbf{P 3}^{\prime}$. The algebraic exceptional set is a proper subset.

P4. If $X$ is non-singular, there exists a pseudo volume form $\Psi$ such that $\operatorname{Ric}(\Psi)>0$.

P5. $X$ is measure hyperbolic. 
Without the pseudo, the problem is whether the following conditions are equivalent:

1. Every subvariety of $X$ (including $X$ itself) is pseudo canonical.

2. $X$ is mordellic.

3. $X$ is hyperbolic.

3'. Every rational map of $\mathbf{P}^{1}$ or an abelian variety into $X$ is constant.

4. If $X$ is non-singular, there exist a positive $(1,1)$-form $\omega$ and a number $B>0$ such that for every complex one-dimensional immersed submanifold $Y$ in $X$,

$$
\operatorname{Ric}(\omega \mid Y) \geqq B \omega \mid Y .
$$

5. Every subvariety of $X$ (including $X$ itself) is measure hyperbolic.

No two properties in each column are known to be equivalent. Some implications are known, and will be discussed at length. In order to make this article as accessible as possible to a broad audience, I reproduce complete proofs for several basic results of Brody, Grauert-Reckziegel, Griffiths, and Kobayashi-Ochiai complemented by Kodaira, proving some of the above implications. But I also survey other matters, with the selections made for their application to certain diophantine questions. Kobayashi's survey [Ko 3] takes other directions. As usual, I would like to remind the reader that a choice made here is not meant to exclude other points of view, and is only dictated by logistical boundary conditions.

Evidence for the diophantine conjectures comes from their self-coherence, rather than special cases. I remind the reader that until Faltings' theorem, there was not known a single example of a curve (variety of dimension 1) which was proved to be mordellic.

Some of the listed conditions, like P1, P2, $\mathbf{P 3}^{\prime}$ and $1,2, \mathbf{3}^{\prime}$ are algebraic. The others are analytic. Different readers can thus be interested in different combinations of them.

In this article, I concentrate on the finiteness of rational points, and I omit the whole area of intermediate hyperbolicity involving holomorphic maps of balls of intermediate dimension-between 1 and the dimension of the manifold-originally defined by Eisenman in his thesis (1969), see [E]. Results analogous to those mentioned here have been obtained, and similar conjectures can be made, especially concerning the dimension and parametrization of the exceptional set discussed in $\S 8$. For an extensive bibliography of papers and a good survey of results on the analytic aspects of this intermediate-dimensional situation, see a forthcoming paper of Graham-Wu [G-W].

The conjectures of this paper concerning mordellic properties are qualitative. I refer the reader to Vojta [Vo] for the extraordinary connection which he established conjecturally between diophantine analysis and the Second Main Theorem of Nevanlinna theory. Vojta's conjectures amount to quantitative estimates for the heights of rational points in the higher-dimensional case, and involve diophantine approximations in an arithmetic-geometric setting. Some of the conjectures of this article can be viewed as providing more information on the sets where Vojta's conjectured inequalities do not hold. 
In addition, parallel to the absolute diophantine questions there has also been progress in higher-dimensional results in the context of sections of algebraic families, e.g. by Kobayashi-Ochiai [K-O 2], Noguchi [No], and Riebesehl [Ri], following Manin and Grauert. I shall mention this work more precisely in an appendix.

I am very grateful to Artin, Friedman, Green, Griffiths, Harris, Siu, Sommese, and Vojta for useful conversations which have helped me sort out the literature and clarify my ideas. I especially thank Green and Griffiths for communicating to me their conjecture in $\S 8$, and explaining to me various matters pertaining to their paper [Gr-Gr].

\section{Contents}

1. Kobayashi hyperbolicity

2. Brody's criterion for hyperbolicity

3. Volume forms; the Ricci form, and the Griffiths function

4. Distance and measure decreasing maps; Ahlfors-Schwarz lemma

5. Pseudo canonical varieties (general type)

6. Minimal models

7. Length functions, ampleness and hyperbolicity

8. Jet differentials

Historical Appendix: algebraic families

\section{§1. Kobayashi hyperbolicity}

Let $\mathbf{D}$ be the open unit disc. The Poincaré metric, also called the hyperbolic metric, on $\mathbf{D}$ is defined by the form

$$
\frac{d z d \bar{z}}{\left(1-|z|^{2}\right)^{2}}
$$

The tangent space $T_{z}(\mathbf{D})$ at a given point $z$ can be identified with $\mathbf{C}$, and if $v \in T_{z}(\mathbf{D})=\mathbf{C}_{z}$ under this identification, then the hyperbolic norm of $v$ under the metric is

$$
|v|_{\text {hyp }, z}=\frac{|v|_{\text {euc }}}{1-|z|^{2}},
$$

where $|v|_{\text {euc }}$ is the ordinary absolute value on $\mathbf{C}$. Instead of $\mathbf{C}$, we write $\mathbf{C}_{z}$ to specify the Poincare metric on $\mathbf{C}$ at the point $z$ of $\mathbf{D}$. Note that for $z=0$, the Poincaré metric is the euclidean metric.

Similarly, for any positive number $r$, we let $\mathbf{D}_{r}$ be the disc of radius $r$, with the Poincaré metric corresponding to the form

$$
\frac{d z d \bar{z}}{\left(1-|z|^{2} / r^{2}\right)^{2}} \text {. }
$$

The hyperbolic norm of a tangent vector $v$ at $z$ is then given by the similar formula as above, replacing $|z|^{2}$ by $|z|^{2} / r^{2}$. 
We shall investigate the behavior of the Poincaré metric under holomorphic maps. We begin with a classical result, at the level of elementary complex variables.

Proposition 1.1 (Schwarz-Pick Lemma). Let $f: \mathbf{D} \rightarrow \mathbf{D}$ be a holomorphic map of the disc into itself. Then

$$
\frac{\left|f^{\prime}(z)\right|}{1-|f(z)|^{2}} \leqq \frac{1}{1-|z|^{2}}
$$

Proof. Fix $a \in$ D. Let

$$
g(z)=\frac{z+a}{1+\bar{a} z} \quad \text { and } \quad h(z)=\frac{z-f(a)}{1-\overline{f(a)} z} .
$$

Thus $g$ and $h$ are automorphisms of the disc which map 0 on $a$ and $f(a)$ on 0 respectively. We let

$$
F=h \circ f \circ g,
$$

so that $F: \mathbf{D} \rightarrow \mathbf{D}$ is holomorphic and $F(0)=0$. Then by the chain rule,

$$
\begin{aligned}
F^{\prime}(0) & =h^{\prime}(f(a)) f^{\prime}(a) g^{\prime}(0) \\
& =\frac{1-|a|^{2}}{1-|f(a)|^{2}} f^{\prime}(a)
\end{aligned}
$$

by a direct computation. By the ordinary Schwarz lemma, we have

$$
\left|F^{\prime}(0)\right| \leqq 1
$$

with equality if and only if $F$ is an automorphism, so $f$ is an automorphism. We also get the reformulation

$$
\frac{\left|f^{\prime}(a)\right|}{1-|f(a)|^{2}} \leqq \frac{1}{1-|a|^{2}},
$$

which proves the proposition.

As already remarked in the proof of the proposition, we have equality at one point if and only if $f$ is an automorphism. In particular, we can express the lemma invariantly in terms of the differential of $f$ as follows.

Let $f: \mathbf{D} \rightarrow X$ be a holomorphic mapping into a complex hermitian manifold. Then we have an induced tangent linear map for each $z \in \mathbf{D}$ :

$$
d f(z): T_{z}(\mathbf{D})=\mathbf{C}_{z} \rightarrow T_{f(z)}(X) .
$$

Each complex tangent space has its norm: $T_{f(z)}$ has the hermitian norm, and $T_{z}(\mathrm{D})=\mathrm{C}_{z}$ has the hyperbolic norm. We can define the norm of the linear map $d f(z)$ as usual:

$$
|d f(z)|=\sup _{v}|d f(z) v| /|v|
$$


for $v \in T_{z}(\mathbf{D}), v \neq 0$. Then the Schwarz-Pick Lemma can be stated in the form:

Corollary 1.2. (i) A holomorphic map $f: \mathbf{D} \rightarrow \mathbf{D}$ is distance decreasing for the hyperbolic norm.

(ii) An automorphism of $\mathbf{D}$ is an isometry.

Unless otherwise specified, the norm on the tangent space $C_{z}$ will be the hyperbolic norm.

Let $X$ be a complex space. Let $x, y \in X$. We consider sequences of holomorphic maps

$$
f_{i}: \mathbf{D} \rightarrow X, \quad i=1, \ldots, m
$$

and points $p_{i}, q_{i} \in \mathbf{D}$ such that $f_{1}\left(p_{1}\right)=x, f_{m}\left(q_{m}\right)=y$, and

$$
f_{i}\left(q_{i}\right)=f_{i+1}\left(p_{i+1}\right) \text {. }
$$

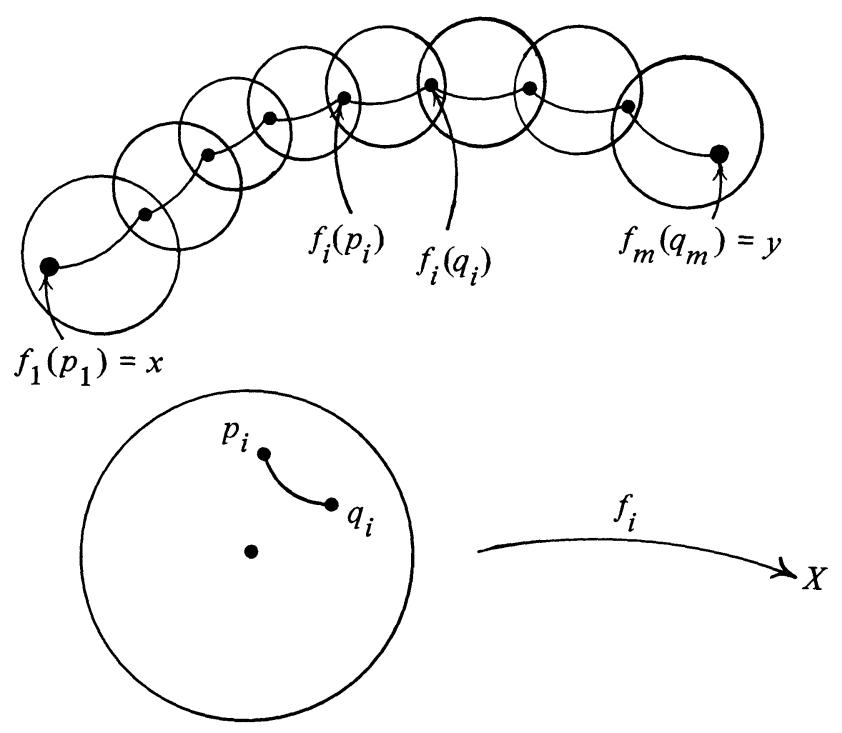

In other words, we join $x$ to $y$ by a chain of discs. We add the hyperbolic distances between $p_{i}$ and $q_{i}$, and take the inf over all such choices of $f_{i}, p_{i}, q_{i}$ to define the Kobayashi semi distance

$$
d_{X}(x, y)=\inf \sum_{i=1}^{m} d_{\text {hyp }}\left(p_{i}, q_{i}\right) .
$$

Then $d_{X}$ satisfies the properties of a distance, except that $d_{X}(x, y)$ may be 0 if $x \neq y$, so $d_{X}$ is a semi distance.

Example 1. If $X=\mathbf{D}$ is the hyperbolic disc, then $d_{X}$ on $X$ coincides with the hyperbolic distance by Corollary 1.2 (the fact that a holomorphic map of $\mathbf{D}$ into itself is distance decreasing). 
Example 2. Let $X=\mathrm{C}$ with the euclidean metric. Then $d_{X}(x, y)=0$ for all $x, y \in \mathbf{C}$. Indeed, given $x \neq y$ there exists a disc of arbitrarily large radius imbedded in $\mathbf{C}$ such that 0 maps to $x$ and $y-x$ maps to $y$. By a dilation, we can map $f: \mathbf{D} \rightarrow \mathbf{C}$ such that $f(0)=x$ and $f(q)=y$ where $q$ is very close to 0 . But the hyperbolic metric is very close to the euclidean metric near 0 , so $d_{X}(x, y)$ is arbitrarily small, so equal to 0 .

It will be useful to consider the following generalization. Let $X$ be a subset of a complex hermitian manifold $Z$. We can define $d_{X}$ on $X$ (rather than on $Z$ ) by taking the maps $f_{i}$ to lie in $X$, and to be holomorphic as maps into $Z$. Then we obtain a semi distance on $X$. We say that $X$ is Kobayashi hyperbolic in $Z$ if this semi distance is a distance, that is if $x \neq y$ implies $d_{X}(x, y) \neq 0$. For simplicity, we shall say hyperbolic instead of Kobayashi hyperbolic. We need $Z$ only to be able to define a holomorphic map of $\mathbf{D}$ into something. From now on, unless otherwise specified, we suppose that we deal with this situation. When we speak of a holomorphic map $f: \mathbf{D} \rightarrow X$ we mean a holomorphic map $f: \mathbf{D} \rightarrow Z$ whose image is contained in $X$.

The foundations of complex analytic spaces actually allow one to define analytic maps from one such space to another. Furthermore, if $X$ is an analytic space imbedded in a complex manifold $Z$, then a map into $X$ is analytic if and only if it is analytic viewed as a map into $Z$. Therefore the definition of the Kobayashi semi distance on $X$ is intrinsic, independent of the imbedding of $X$ into a manifold.

We shall also consider hermitian metrics, and for this it is useful to have the analytic space imbedded in a manifold so that we can use norms on a tangent space (which does not exist when $X$ has singularities). For instance in Theorem 2.2 below, we give Brody's criterion for a compact subspace of a complex hermitian manifold to be hyperbolic. Without mentioning the norms, this result will imply the following statement.

Let $X$ be a compact analytic space. Then $X$ is hyperbolic if and only if every holomorphic map $f: \mathbf{C} \rightarrow X$ is constant.

In this context, it is useful to define an analytic space $X$ to be Brody hyperbolic if every holomorphic map of $\mathbf{C}$ into $X$ is constant. The above statement then can be rephrased to say that for a compact analytic space, Brody hyperbolic is equivalent with Kobayashi hyperbolic. Let $f: \mathbf{D} \rightarrow X$ be a holomorphic map. In line with Royden's approach [Ro] we define

$$
c(f)=\sup _{z}|d f(z)|
$$

where the sup is taken over all $z \in \mathbf{D}$. We also define

$$
c(X)=\sup _{f} c(f) \text {, }
$$

where the sup is taken over holomorphic maps $f: \mathbf{D} \rightarrow X$ (so, according to our conventions, holomorphic maps $f: \mathbf{D} \rightarrow Z$ whose image is contained in $X$ ). We do not exclude that $c(f)$ and $c(X)$ are equal to $\infty$. Since, however, 
automorphisms of the disc operate transitively on the disc, it follows that we have

$$
c(X)=\sup _{f}|d f(0)|,
$$

where the sup is taken as before. Obviously $c(f) \leqq c(X)$.

Lemma 1.3. Let $f: \mathbf{D} \rightarrow X$ be holomorphic. Let $z_{1}, z_{2} \in \mathbf{D}$. Then

$$
d_{\text {her }}\left(f\left(z_{1}\right), f\left(z_{2}\right)\right) \leqq c(f) d_{\text {hyp }}\left(z_{1}, z_{2}\right) \leqq c(X) d_{\text {hyp }}\left(z_{1}, z_{2}\right) \text {. }
$$

Proof. Let $\gamma:[0,1] \rightarrow \mathbf{D}$ be a geodesic between $z_{1}$ and $z_{2}$ in $\mathbf{D}$. Then $f \circ \gamma$ is a curve joining $f\left(z_{1}\right)$ and $f\left(z_{2}\right)$ and its length is

$$
\int_{0}^{1}\left|d f(\gamma(t)) \gamma^{\prime}(t)\right|_{\text {her }} d t \leqq c(f) \int_{0}^{1}\left|\gamma^{\prime}(t)\right|_{\text {hyp }} d t,
$$

so length of $f \circ \gamma \leqq c(f)$ length of $\gamma$, whence the lemma follows.

Theorem 1.4 (Brody [Br]). If $c(X)$ is finite then $X$ is hyperbolic.

Conversely, if $X$ is compact and hyperbolic then $c(X)$ is finite.

Proof. Suppose $c(X)$ finite. By Lemma 1.3 for $x \neq y$ in $X$ we obtain

$$
d_{X}(x, y) \geqq(1 / c) d_{\text {herm }}(x, y)>0,
$$

whence the first part of the theorem follows.

Conversely, suppose $c(X)=\infty$ and $X$ compact. There exists a sequence of holomorphic maps

$$
f_{n}: \mathbf{D} \rightarrow X
$$

with $\left|d f_{n}(0)\right|$ increasing to $\infty$. By compactness, say $\lim f_{n}(0)=x$. Take a chart at $x$

$$
U \subset U^{\mathrm{cl}} \subset V,
$$

where $U, V$ are open balls, say, centered at 0 , and $U^{\mathrm{cl}}$ is the closure of $U$. Let $S$ be the boundary of $U$ and let $s$ be the radius of $S$. Let $r<1$ be a positive number such that $f_{n}\left(\mathbf{D}_{r}^{\text {cl }}\right) \subset U$. Then

$$
f_{n}^{\prime}(0)=\frac{1}{2 \pi i} \int_{C_{r}} \frac{f_{n}(w)}{w^{2}} d w \quad \text { so } \quad\left|f_{n}^{\prime}(0)\right| \leqq s / r .
$$

Since the hyperbolic metric at $z=0$ is the same as the euclidean metric, it follows that if $\left|f_{n}^{\prime}(0)\right|$ is large, then $r=r(n)$ has to tend to 0 . Therefore, given a positive integer $m$, there exists $n$ such that $f_{n}\left(\mathbf{D}_{1 / m}\right)$ is not contained in $U$ and therefore intersects the boundary $S$. Hence we can find a point $x_{m}$ on $S$ such that $x_{m}=f_{n}\left(p_{m}\right)$ with $p_{m} \in \mathbf{D}_{1 / m}$. Then

$$
d_{X}\left(x, x_{m}\right)=d_{X}\left(f_{n}(0), f_{n}\left(p_{m}\right)\right) \leqq d_{\text {hyp }}\left(0, p_{m}\right) \rightarrow 0
$$

as $m \rightarrow \infty$. But a subsequence of $x_{m}$ converges to a point $y \in S$ so $d_{X}(x, y)$ $=0$ and $X$ is not hyperbolic. This proves the theorem. 
In the compact case, Brody's criterion can be taken as a definition. Similarly, Theorem 2.2 in the next section will give another important equivalent condition. Thus from the start, we have three valuable characterizations of hyperbolic varieties, which exist on an equal footing. The Kobayashi condition and Theorem 1.4 put special emphasis on mapping discs into the manifold.

Remark 1. This context also allows transposing certain questions from the theory of transcendental numbers. For instance if $f: \mathbf{D} \rightarrow X$ is a holomorphic immersion into a hyperbolic variety $X$ defined over a number field, if $d f(0)$ is algebraic, and $f(0)$ is an algebraic point, then is $f\left(z_{0}\right)$ transcendental for $z_{0}$ algebraic $\neq 0$ ? Is the radius of the disc transcendental? See [La 3], and results of Wolfart [Wo], Wolfart-Wustholz [Wo-Wu] for special Riemann surfaces, relating the radius to special values of the gamma (beta) function which also arise as periods.

Remark 2. Instead of using the unit disk and having $|d f(0)| \rightarrow \infty$, in the next section we shall use $|d f(0)|=1$, say, with discs of increasing radius. If the variety is hyperbolic, then this radius is bounded. For Riemann surfaces, this is the classical Landau-Schottky theorem, and a variation is due to Bloch. In this article dealing with the higher-dimensional case, I give one formulation of the generalizations in terms of the Schwarz lemma. For the other possible formulations and variants, explaining the historical motivation, see Griffiths [Gri]. One can again ask for the arithmetic nature (including transcendence) of the sup of radii $r$ such that there is a holomorphic map

$$
f: \mathbf{D}_{r} \rightarrow X
$$

into a hyperbolic variety with $|d f(0)|=1$. This supcould be called the Landau-Schottky constant associated with the metric.

Similarly, one can ask for the arithmetic nature of the constant $c(X)$, which also depends on the metric.

Remark 3. Consider a holomorphic curve $f: \mathbf{D} \rightarrow X$ into a hyperbolic variety. The finiteness of rational points in a field $F$ amounts to the property that if $\mathbf{D}_{r}$ is a closed subdisc, then there are only a finite number of values $z \in \mathbf{D}_{r}$ such that $f(z) \in X(F)$. In the theory of transcendental numbers, one considers such parametrizations, proving the transcendence of a uniformization map $f$ at certain points. But Siegel in some cases also proved that the images of certain points if algebraic must have a high degree [Si]. I have had the impression for a long time that the methods using approximating functions should apply to this case to yield the finiteness.

Remark 4. Using $\mathbf{C}_{p}$ instead of $\mathbf{C}$ and the theory of rigid analytic spaces, it should be possible to translate $p$-adically the various characterizations of hyperbolicity. It then becomes a problem to determine the relations between the various possible definitions of $p$-adic hyperbolicity, and to relate these analytic properties with the algebraic characterization of Conjecture 5.6 and the diophantine properties listed in $\$ 5$. 


\section{§2. Brody's criterion for hyperbolicity}

If $X$ is hyperbolic, then directly from the definition of the Kobayashi pseudo metric and Example 2 of $\$ 1$, there cannot be a non-constant holomorphic map $f: \mathbf{C} \rightarrow X$. The converse is due to Brody [Br], and is the main goal of this section. We start with:

Lemma 2.1 (Brody's reparametrization lemma). Let $X$ be a subset of a complex hermitian manifold. Let $f: \mathbf{D}_{r} \rightarrow X$ be holomorphic. Let $c>0$ and for $0 \leqq t \leqq 1$ let $f_{t}(z)=f(t z)$.

(i) If $|d f(0)|>c$ then there exists $t<1$ and an automorphism $h$ of $\mathbf{D}$ such that if we let

then

$$
g=f_{t} \circ h
$$

$$
\sup _{z \in \mathbf{D}_{r}}|d g(z)|=|d g(0)|=c .
$$

(ii) If $|d f(0)|=c$, then we get the same conclusion allowing $t \leqq 1$.

Proof. Let $m_{t}: \mathbf{D}_{r} \rightarrow \mathbf{D}_{r}$ be multiplication by $t$, so that $f_{t}$ can be factored:

$$
\mathbf{D}_{r} \stackrel{m_{t}}{\rightarrow} \mathbf{D}_{r} \stackrel{f}{\rightarrow} X
$$

Then $d m_{t}(z) v=t v$, so

$$
\left|d f_{t}(z)\right|=|d f(t z)| t \frac{1-|z|^{2} / r^{2}}{1-|t z|^{2} / r^{2}} .
$$

Let

$$
s(t)=\sup _{z \in \mathbf{D}_{r}}\left|d f_{t}(z)\right|
$$

Then $s(t)$ is increasing for $0 \leqq t \leqq 1$. Indeed, if $t_{1}<t_{2}<1$ and $z_{1}$ is such that

$$
s\left(t_{1}\right)=\left|d f_{t_{1}}\left(z_{1}\right)\right|,
$$

then we let $z_{2}=t_{1} z_{1} / t_{2}$ so that $t_{2} z_{2}=t_{1} z_{1}$. A simple comparison of the extra factor then shows that

$$
\left|d f_{t_{1}}\left(z_{1}\right)\right|<\left|d f_{t_{2}}\left(z_{2}\right)\right| \leqq s\left(t_{2}\right)
$$

If $t<1$ then the extra factor

$$
t \frac{r^{2}-|z|^{2}}{r^{2}-|t z|^{2}} \text { is less than } 1,
$$

and if $t=1$ then this extra factor is equal to 1 , so $s(t)$ is also increasing up to $t=1$. Also we can write $t z=w$. Taking the sup for $z \in \mathbf{D}_{r}$ amounts to taking the sup for $w \in t \mathbf{D}_{r}$. If $t<1$, we can even take the sup over the closure $t \mathbf{D}_{r}^{\mathrm{cl}}$. It follows that $s(t)$ is continuous for $0 \leqq t<1$. Also $s(t) \rightarrow s(1)$ as $t \rightarrow 1$, even if $s(1)=\infty$. By assumption in the first part, $|d f(0)|>c$, and hence $s(1)>c$. 
Hence there exists $0 \leqq t<1$ such that $s(t)=c$. Hence there is some $z_{0} \in t \mathbf{D}_{r}^{\text {cl }}$ such that $\left|d f_{t}\left(z_{0}\right)\right|=c$. Now let $h: \mathbf{D}_{r} \rightarrow \mathbf{D}_{r}$ be the automorphism such that $h(0)=z_{0}$ and let $g=f_{t} \circ h$. Then

$$
|d g(0)|=\left|d f_{t}\left(z_{0}\right)\right||d h(0)|=\left|d f_{t}\left(z_{0}\right)\right|=c,
$$

thus proving the first part. The second part is proved similarly, allowing $t=1$.

Theorem $2.2[\mathrm{Br}]$. Let $X$ be a relatively compact complex subspace of a complex hermitian manifold, and suppose $X$ is not hyperbolic. Then there exists a non-constant holomorphic map $f: \mathbf{C} \rightarrow X^{\mathrm{cl}}$ such that

$$
|d f(z)|_{\text {euc }} \leqq 1 \quad \text { for all } z \in \mathbf{C} \text { and }|d f(0)|=1 .
$$

In particular, the hermitian area of $f\left(\mathbf{D}_{r}\right)$ is $\leqq \pi r^{2}$.

Remark. Since $f: \mathbf{C} \rightarrow X$ has domain $\mathbf{C}$, we are using the euclidean hermitian metric on $\mathbf{C}$, so the norm of $d f(z)$ is now measured as going from the euclidean norm to the hermitian norm in $X$. This is the reason for putting the subscript on this norm in the statement of the theorem.

Proof. By Theorem 1.4, there exists a sequence of maps

$$
f_{n}: \mathbf{D} \rightarrow X \text { such that }\left|d f_{n}(0)\right| \rightarrow \infty .
$$

Without loss of generality, making a dilation, we may consider a sequence of maps

$$
f_{n}: \mathbf{D}_{r_{n}} \rightarrow X \text { such that }\left|d f_{n}(0)\right|=1
$$

and the radii $r_{n}$ increase to infinity. By Lemma 2.1 there exist holomorphic maps

$$
g_{n}: \mathbf{D}_{r_{n}} \rightarrow X \quad \text { such that }\left|d g_{n}(0)\right|=1=\sup _{z \in \mathbf{D}_{r_{n}}}\left|d g_{n}(z)\right|
$$

We want to show that given a compact subset $K$ of $\mathbf{C}$ there exists a subsequence of $\left\{g_{n}\right\}$ which converges uniformly on $K$. This is a simple matter, based on the following arguments.

Let $z_{0} \in \mathbf{C}$. By compactness it suffices to prove that there is a neighborhood of $z_{0}$ on which a subsequence converges uniformly. After passing to a subsequence, we may assume without loss of generality that $\left\{g_{n}\left(z_{0}\right)\right\}$ converges to a point $x_{0}$ of $X$. Now pick a chart at $x_{0}$, say an open set $V$ and a holomorphic isomorphism

$$
\varphi: V \rightarrow \mathbf{B}_{s},
$$

where $\mathbf{B}_{s}$ is the open ball of radius $s$ centered at 0 . We may assume that the closure of $V$ is contained in another chart, isomorphic to a ball of bigger radius. Then by hypothesis, there is a positive number $C$ such that

$$
\left|\left(\varphi \circ g_{n}\right)^{\prime}(z)\right| \leqq C \quad \text { for all } z \in g_{n}^{-1}\left(V^{\mathrm{cl}}\right) \text { and all } n \text {. }
$$




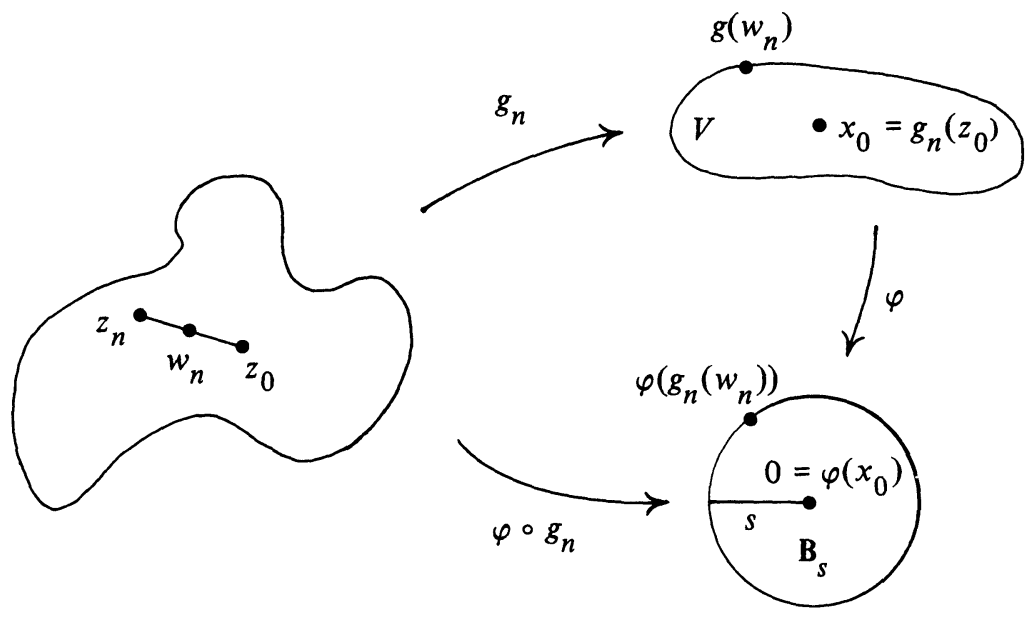

The arguments which follow are concerned with this simple situation: We have a sequence $g_{n}: U \rightarrow X$ of holomorphic maps of some open $U$ in $\mathbf{C}$ into $X$, a point $z_{0} \in U$ such that $g_{n}\left(z_{0}\right)$ converges to $x_{0}$, and charts as above, such that the derivative $\left(\varphi \circ g_{n}\right)^{\prime}$ is bounded on $g_{n}^{-1}\left(V^{\mathrm{cl}}\right)$. We now prove equicontinuity statements for this situation.

1. There exists a disc $\mathbf{D}_{a}\left(z_{0}\right)$ such that $g_{n}\left(\mathbf{D}_{a}\right) \subset V$ for all $n$, that is $\mathbf{D}_{a}\left(z_{0}\right) \subset g_{n}^{-1}(V)$ for all $n$.

Proof. Otherwise, there exists a sequence $\left\{z_{n}\right\}$ tending to $z_{0}$ such that $g_{n}\left(z_{n}\right) \notin V$ for arbitrarily large $n$. On the segment from $z_{0}$ to $z_{n}$, let $w_{n}$ be the first point such that $g_{n}\left(w_{n}\right)$ lies on the boundary of $V$, so $\varphi \circ g_{n}\left(w_{n}\right)$ lies on the sphere bounding $\mathbf{B}_{s}$. Then

$$
\left(\varphi \circ g_{n}\right)\left(w_{n}\right)=\int_{z_{0}}^{w_{n}}\left(\varphi \circ g_{n}\right)^{\prime}(\zeta) d \zeta
$$

where the integral is taken over the line segment between $z_{0}$ and $w_{n}$. Thus we obtain the bound

$$
\left|\varphi \circ g_{n}\left(w_{n}\right)\right| \leqq\left|w_{n}-z_{0}\right| C .
$$

As $n \rightarrow \infty$, the left side approaches $s$ and the right side approaches 0 , a contradiction.

2. The sequence $\left\{g_{n}\right\}$ is equicontinuous at $z_{0}$. In fact for $z, z^{\prime} \in \mathbf{D}_{a}\left(z_{0}\right)$ (where $\mathbf{D}_{a}\left(z_{0}\right)$ is the disc as in the previous statement), we have for all $n$ :

$$
\left|\varphi \circ g_{n}(z)-\varphi \circ g_{n}\left(z^{\prime}\right)\right| \leqq\left|z-z^{\prime}\right| C .
$$

Proof. Immediate from the integral formula, which can now be applied uniformly for all $n$. 
This proves that the sequence $\left\{g_{n}\right\}$ converges uniformly on compact sets, by Ascoli's theorem.

Going back to Theorem 2.2 proper, we get a subsequence of $\left\{g_{n}\right\}$ converging uniformly on $\mathbf{D}_{1}$ to a map $f$. A further subsequence converges uniformly on $\mathbf{D}_{2}$, and so on. We can then extend $f$ analytically to all of $\mathbf{C}$. Furthermore $f$ is not constant since

$$
|d f(0)|=\lim _{n \rightarrow \infty}\left|d f_{n}(0)\right|=1 .
$$

Finally, consider the euclidean metric on $\mathbf{D}_{r} \subset \mathbf{C}$, together with the holomorphic map $f: \mathbf{C} \rightarrow X$ just obtained. The tangent map

$$
d f(z): T_{z}(\mathbf{C})=\mathbf{C} \rightarrow T_{f(z)}
$$

is a linear map with euclidean norm on $T_{z}(\mathbf{C})$ and hermitian norm on $T_{f(z)}$ (of the ambient manifold). Let $z_{0} \in \mathbf{C}$. Then

$$
\left|d f\left(z_{0}\right)\right|_{\text {euc }}=\lim _{n \rightarrow \infty}\left|d g_{n}\left(z_{0}\right)\right|_{\text {euc }} \leqq \lim _{n \rightarrow \infty}\left(1-\left|z_{0}\right|^{2} / r_{n}^{2}\right)^{-1}=1 \text {. }
$$

Finally, the area of $f\left(\mathbf{D}_{r}\right)$ is bounded by the euclidean area of $\mathbf{D}_{r}$, which is $\pi r^{2}$. This concludes the proof of the theorem.

I once made the conjecture [La 1]:

Conjecture 2.3. Let $X$ be a projective variety. If $X$ is hyperbolic, then $X$ is mordellic.

To make the conjecture imply that a subvariety of an abelian variety has only a finite number of rational points unless these are contained in translated abelian subvarieties, I conjectured that a subvariety of an abelian variety which does not contain translations of abelian subvarieties $\neq 0$ is hyperbolic. This conjecture was proved by Mark Green [Gr] as follows.

Theorem 2.4. Let $X$ be a closed subset of a complex torus $\mathbf{T}$, with standard hermitian metric induced from a representation $\mathbf{T}=\mathbf{C}^{d} / \Lambda$, where $\Lambda$ is a lattice. Assume also that $X$ is an analytic subvariety, that is $X$ is equal to its complex analytic closure. Then $X$ is hyperbolic if and only if $X$ does not contain a translated complex subtorus $\neq 0$.

Proof. A complex subtorus in $X$ would give a complex line $\mathbf{C} \rightarrow X$ which would contradict Brody's Theorem 2.2.

Conversely, if $X$ is not hyperbolic, then Brody's theorem yields a holomorphic map $f: \mathbf{C} \rightarrow X$ such that $|d f(z)| \leqq 1$ and $|d f(0)|=1$ (euclidean norms). Then $f$ lifts to a holomorphic map into the universal covering space of $\mathbf{T}$, making the following diagram commutative:

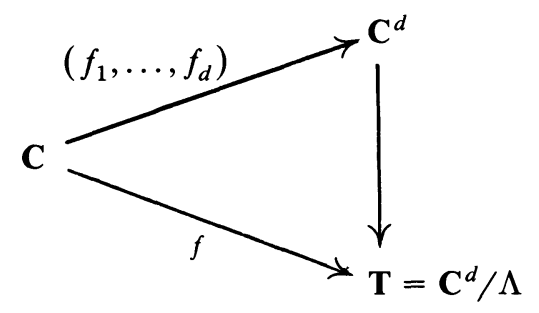


and we have $\sum_{i=1}^{d}\left|f_{i}^{\prime}\right|^{2} \leqq 1$. Hence $f_{i}^{\prime}$ is constant for all $i$ by Liouville's theorem, so $f_{i}$ is linear. After a translation of $X$ we may assume without loss of generality that $f(0)=0$, and $f$ is a one-parameter subgroup. Since $X$ is assumed to be a complex analytic variety, the complex analytic closure of the image of $f$ is itself a complex analytic group, which is therefore a complex subtorus contained in $X$. This concludes the proof.

Remark. We have used an elementary lemma:

Let $G$ be a group with a topology (not necessarily Hausdorff, since in the applications it may be a Zariski topology). Assume that given $x \in G$, translation by $x$ is bicontinuous, and also that $x \mapsto x^{-1}$ is bicontinuous. Let $H$ be an abstract subgroup of $G$. Then the closure $\bar{H}$ is a subgroup.

Proof. The closure of a subset $S$ is the intersection of all closed subsets of $G$ containing $S$. Since $x \mapsto x^{-1}$ is bicontinuous, it follows that $\bar{H}^{-1}$ is a closed set containing $H^{-1}$, so

$$
\bar{H}=\operatorname{closure}(H)=\operatorname{closure}\left(H^{-1}\right) \subset \bar{H}^{-1} .
$$

Applying the inverse map to this inclusion yields $\bar{H}^{-1} \subset \bar{H}$, so

$$
\bar{H}=\bar{H}^{-1} \text {. }
$$

Next let $h \in H$. Then $h H \subset \bar{H}$, so $H \subset h^{-1} \bar{H}$, which is closed, so $\bar{H} \subset h^{-1} \bar{H}$, whence finally $h \bar{H} \subset \bar{H}$. Therefore $H \bar{H} \subset \bar{H}$. Similarly, $\bar{H} H \subset \bar{H}$. Finally, let $h \in \bar{H}$. Then $h H \subset \bar{H}$ by what we have just proved, and hence $H \subset h^{-1} \bar{H}$ so $\bar{H} \subset h^{-1} \bar{H}$ so finally $h \bar{H} \subset \bar{H}$. So we have proved

$$
\bar{H} \bar{H} \subset \bar{H} \text {. }
$$

This concludes the proof of the lemma.

Note that we did not assume $G$ to be a topological group because, in applications, the composition map $G \times G \rightarrow G$ is not continuous. Only translation by single elements is bicontinuous.

We give another application also due to Green [Gr].

Lemma 2.5. Let $\mathbf{T}$ be a complex torus, and let $X$ be an effective divisor on $\mathbf{T}$, not containing non-trivial translated complex subtoruses. If $T-X$ is not hyperbolic, then there exists a translation of a one-parameter group

$$
g: \mathbf{C} \rightarrow \mathbf{T}-X
$$

Proof. As in Theorem 2.2, there exists a sequence of holomorphic maps

$$
g_{n}: \mathbf{D}_{r_{n}} \rightarrow \mathbf{T}-X
$$

with $r_{n} \rightarrow \infty$ and such that

$$
\left|d g_{n}(0)\right|=1=\sup _{z \in \mathbf{D}_{r_{n}}}\left|d g_{n}(z)\right| \text {. }
$$

By a diagonal selection, after picking subsequences we may assume without loss of generality that $\left\{g_{n}\right\}$ converges uniformly on compact subsets of $\mathbf{C}$ (each compact set is contained in $\mathbf{D}_{r_{n}}$ for $n$ sufficiently large), and the convergence is 
to an analytic function

$$
g: \mathbf{C} \rightarrow \mathbf{T} .
$$

The problem is now whether the image of $g$ can intersect $X$.

If $g(\mathbf{C})$ is contained in $X$ then $g$ is constant by Theorem 2.4 and Theorem 2.2 , but this is not possible since $|d g(0)|=\lim \left|d g_{n}(0)\right|$. Hence $g(\mathbf{C})$ is not contained in $X$. Suppose that $g(\mathbf{C})$ intersects $X$. We first prove that $g^{-1}(X)$ consists of isolated points in C. Suppose $X$ is defined locally on an open set $V$ by a function $\varphi=0$. Let $x \in X \cap V$ and let $U$ be a connected component of $g^{-1} V$. Then $\varphi \circ g$ is a holomorphic function of $U$ into $\mathrm{C}$ and hence its zeros are isolated or this function is the constant $\varphi(x)=0$. If $\varphi \circ g(U)=0$ this means that $g(U) \subset X$. Suppose this is the case. Let $U^{\prime}$ be a small disc such that $g\left(U^{\prime}\right)$ is contained in a chart where $X$ is defined by one equation $\varphi^{\prime}=0$, and such that $U \cap U^{\prime}$ is not empty. Then $\varphi \circ g\left(U \cap U^{\prime}\right)=0$, and hence by analytic continuation, $g\left(U^{\prime}\right)$ is contained in $X$. In this way, we can see that every compact disc in $\mathbf{C}$ is mapped into $X$ by $g$, and so $g(\mathbf{C}) \subset X$, which is a contradiction.

It then follows that $g^{-1}(X)$ consists of isolated points in C. We shall now prove that $g(\mathbf{C})$ does not intersect $X$. Suppose there is some $z_{0} \in \mathbf{C}$ such that $g\left(z_{0}\right) \in X$. Let $S$ be a small circle centered at $z_{0}$ such that $g(S) \subset \mathbf{T}-X$. Such a circle exists since $g^{-1}(X)$ consists of isolated points in C. We pick $S$ small enough that $g(S) \subset V$, where $V$ is a small open set containing $g\left(z_{0}\right)$, in which $x$ is defined by the equation $\varphi=0$. Let the winding number

$$
W(\varphi \circ g(S), 0)=\text { number of zeros of } \varphi \circ g \text { inside } S
$$

and

$$
W\left(\varphi \circ g_{n}(S), 0\right)=0 \text { because } g_{n}\left(\mathbf{D}_{r_{n}}\right) \text { does not intersect } X \text {. }
$$

Then

$$
0=\lim _{n \rightarrow \infty} W\left(\varphi \circ g_{n}(S), 0\right)=W(\varphi \circ g(S), 0),
$$

so $\varphi \circ g$ has no zeros inside $S$, whence $g^{-1}(X) \cap \operatorname{Int}(S)$ is empty, contradicting the hypothesis that $g\left(z_{0}\right) \in X$. This proves that

$$
g(\mathbf{C}) \subset \mathbf{T}-X \text {. }
$$

By Theorem 2.2 we have $|d g(z)| \leqq 1$ for all $z \in \mathbf{C}$ and, as in Theorem 2.4 , we conclude that $g$ is the translation of a one-parameter subgroup in $T$. This proves the theorem.

We recall that for an algebraic complex torus, that is, an abelian variety, if $X$ does not contain a translated abelian subvariety of dimension $>0$ then $X$ is ample. Cf. my Abelian Varieties, Chapter IV, \$2. The converse is false. For instance, the Jacobian of a curve which is a quadratic extension of an elliptic curve, of genus 3, ramified at infinity (in the Weierstrass form) has a theta divisor which contains an isogeneous image of the elliptic curve by pull-back. 
Sometimes (see the end of Weil's thesis), people are not aware of this possibility. When it happens, the theta divisor contains infinitely many rational points in some finite extension of a field of definition.

Theorem 2.6. Let $\mathbf{T}$ be a complex torus imbedded in projective space. Let $X$ be an effective divisor which does not contain a translated abelian subvariety of dimension $>0$. Then $\mathbf{T}-X$ is hyperbolic.

Proof. Suppose $\mathbf{T}-X$ is not hyperbolic. After replacing $X$ by a suitable multiple, we may assume without loss of generality that $X$ is very ample, so is a hyperplane section in some projective imbedding. In Lemma 2.5 , let $\mathbf{T}^{\prime}$ be the Zariski closure of $g(\mathbf{C})$. Then $\mathbf{T}^{\prime}$ is a complex subtorus (abelian subvariety), and $X \cap \mathbf{T}^{\prime}=X^{\prime}$ is a hyperplane section of $\mathbf{T}^{\prime}$ in this imbedding. In particular, $X \cap \mathbf{T}^{\prime}$ is not empty. The fact that $g(\mathbf{C})$ is contained in $\mathbf{T}^{\prime}-X^{\prime}$ now contradicts Theorem 3 of [Ax], which implies that if a holomorphic curve in a projective variety is Zariski dense, then the intersection with a hyperplane is not empty, and is infinite if and only if the curve is not algebraic. This concludes the proof.

Remark 1. Green's theorem gives an example when both the compact $X$ and the non-compact $\mathbf{T}-X$ are hyperbolic. Note that $\mathbf{T}-X$ may have infinitely many rational points, while Conjecture 2.3 says that $X$ cannot. Green's original proof of Theorem 2.6 did not rely on Ax's theorem, and proved somewhat more concerning the hyperbolic imbedding of $\mathbf{T}-X$ in $\mathbf{T}$. The variation of the proof given here is also due to Green (oral communication).

Observe that the argument used to prove Lemma 2.5 for the most part has nothing to do with complex toruses, and in fact proves the following statement:

Let $Z$ be a variety and let $X$ be a proper algebraic subset. Assume that $X$ is Brody hyperbolic. If $Z-X$ is Brody hyperbolic, then $Z-X$ is Kobayashi hyperbolic.

Remark 2. I had conjectured Ax's result via the theory of transcendental numbers, in the context of analytic parametrizations. This shows another interrelation between number theory and complex differential-algebraic geometry.

The last two theorems of Green give examples of hyperbolic manifolds, including a non-compact case. Brody and Green give other examples of non-singular hypersurfaces which are deformations of the Fermat surface [Br-Gr]. The Fermat surface is not hyperbolic since it contains lines. The Brody-Green example is the variety $X_{t}$ given by the equation

$$
z_{0}^{d}+z_{1}^{d}+z_{2}^{d}+z_{3}^{d}+\left(t z_{0} z_{1}\right)^{d / 2}+\left(t z_{0} z_{2}\right)^{d / 2}=0
$$

where $d$ is even $\geqq 50$. Thus $X_{0}$ is the Fermat surface, which is non-singular, and $X_{t}$ is a non-singular hypersurface for small $t$. Recall that all non-singular hypersurfaces of the same degree are $C^{\infty}$-isomorphic, but of course not complex analytically isomorphic. Furthermore, such hypersurfaces are simply connected in dimension $\geqslant 2$. Brody and Green show that $X_{t}$ is hyperbolic for 
all small $t \neq 0$. Thus we have three classes of hyperbolic varieties:

(i) Compact quotients of bounded domains by a discrete group operating freely.

(ii) Subvarieties of abelian varieties not containing translated subtoruses of dimension $>0$.

(iii) The Brody-Green family of perturbations of Fermat hypersurfaces.

In this connection, it is worth while to note that Brody [Br] showed that the space of hyperbolic complex structures on a given $C^{\infty}$ manifold is open for the ordinary topology. I do not understand clearly the extent to which hyperbolicity is open for the Zariski topology. However, since a neighborhood of a point for the ordinary topology contains a generic point over some field of definition $F_{0}$, if one had an algebraic characterization of hyperbolicity, it would follow that when a special member of a family is hyperbolic, then the generic member of that family is also hyperbolic.

\section{Pseudo-hyperbolicity.}

As mentioned in the introduction, we can pseudofy hyperbolicity. Let $X$ be a variety. Kiernan and Kobayashi [K-K] discuss the notion of $X$ being hyperbolic modulo a subset $Y$, meaning that the Kobayashi semi distance in $X$ satisfies $d_{X}(x, y) \neq 0$ unless $x=y$ or $x, y \in Y$. In the present system, I would define $X$ to be pseudo Brody hyperbolic if the exceptional $\operatorname{set} \operatorname{Exc}(X)$ is a proper subset; and $X$ is pseudo Kobayashi hyperbolic if there exists a proper algebraic subset $Y$ such that $X$ is hyperbolic modulo $Y$. It is not known if the two definitions are equivalent, although I would certainly conjecture that they are, with $Y=\operatorname{Exc}(X)$. In fact, it is likely that the following should be true:

Let $x \neq y$ be distinct points such that $d_{X}(x, y)=0$. Then there exists a non-constant holomorphic map $f: \mathbf{C} \rightarrow X$ such that $y$ lies in the ordinary closure of $f(\mathbf{C})$, and similarly for $x$.

I have tried to make Brody's argument work to prove this, but without success.

In analogy with Green's Theorem 2.6 , I would make the:

Conjecture 2.7. The complement of the exceptional set is hyperbolic.

In the introduction, we defined the algebraic exceptional set $\operatorname{Exc}_{\text {alg }}(X)$. To make the terminology functorial with respect to the ideas, we could define $X$ to be algebraically hyperbolic if every rational map of $\mathbf{P}^{1}$ or an abelian variety into $X$ is constant. (Actually, since $\mathbf{P}^{1}$ is a rational image of an elliptic curve, we could avoid mentioning $\mathbf{P}^{1}$ in this definition.) Then we say that $X$ is pseudo algebraically hyperbolic if the algebraic exceptional set is a proper subset. Conjecturally, all these pseudo conditions are equivalent. Until the equivalences are proved, one can make various subsidiary conjectures, for instance that the complement of the algebraic exceptional set is hyperbolic.

\section{§3. Volume forms; the Ricci (Chern) form and the Griffiths function}

We shall be interested in one-dimensional manifolds to the extent that they shed light on the higher-dimensional case, in many ways. We give here basic definitions of the "curvature" to be used. 
Let $X$ be a complex manifold and let $L$ be a line bundle. A metric $\rho$ on $L$ consists in giving a norm on each fiber, varying smoothly. If $U$ is the open set of a (coordinate) chart, and $s$ is a section represented by the function $s_{U}$ on $U$, then the norm is represented by a function $\rho_{U}$ such that

$$
|s|^{2}=\left|s_{U}\right|^{2} / \rho_{U}
$$

where $\left|s_{U}\right|$ is the ordinary absolute value.

As usual, we let

$$
d=\partial+\bar{\partial} \quad \text { and } \quad d^{c}=\frac{1}{2 \pi \sqrt{-1}} \frac{1}{2}(\partial-\bar{\partial}) .
$$

Then

$$
d d^{c}=\frac{\sqrt{-1}}{2 \pi} \partial \bar{\partial}
$$

We define the Chern form of the metric to be the real $(1,1)$-form

$$
c_{1}(\rho)=-d d^{c} \log |s|^{2}=d d^{c} \log \rho_{U} \quad \text { on } U .
$$

Let $\Psi$ be a volume form on $X$. This is the same as a metric on the canonical line bundle $K_{X}$. In terms of complex coordinates $z_{1}, \ldots, z_{n}$, such a form is one which can be written

$$
\Psi(z)=\rho(z) \Phi(z) \quad \text { where } \Phi(z)=\prod_{j=1}^{n} \frac{\sqrt{-1}}{2 \pi} d z_{j} \wedge d \bar{z}_{j},
$$

and $\rho$ is real $>0$. In practice one often has

$$
\rho(z)=\sigma(z)|g(z)|^{2},
$$

where $g$ is holomorphic invertible, and $\sigma$ is real $>0$. We define the Ricci form of $\Psi$ to be the Chern form of this metric on $K_{X}$, so that

$$
\operatorname{Ric}(\Psi)=c_{1}(\Psi)=d d^{c} \log \rho=d d^{c} \log \sigma .
$$

Since a holomorphic change of charts changes the volume form precisely by a factor $g \bar{g}$ where $g$ is holomorphic, and since

$$
d d^{c} \log (g \bar{g})=0,
$$

it follows that $\operatorname{Ric}(\Psi)$ is independent of the choice of complex coordinates, and defines a real $(1,1)$-form. In important cases, this real $(1,1)$-form will be positive, but we do not yet assume this. (By positive, we mean strictly positive throughout.)

Remark. If $C$ is a constant, then

$$
\operatorname{Ric}(C \Psi)=\operatorname{Ric}(\Psi) .
$$

If $u$ is a positive function, then

$$
\operatorname{Ric}(u \Psi)=\operatorname{Ric}(\Psi)+d d^{c} \log u .
$$

Both assertions are trivial from the definition.

A 2-form commutes with all forms. By the $n$th power

$$
\operatorname{Ric}(\Psi)^{n}
$$


we mean the $n$th exterior power. Then $\operatorname{Ric}(\Psi)^{n}$ is an $(n, n)$-form, and in particular a top degree form on $X$. Since $\Psi$ is a volume form, there is a unique function $G$ on $X$ such that

$$
\frac{1}{n !} \operatorname{Ric}(\Psi)^{n}=G \Psi .
$$

We may also write symbolically

$$
G=\frac{1}{n !} \operatorname{Ric}(\Psi)^{n} / \Psi .
$$

We call $G$ the Griffiths function, associated with the original $\Psi$, so we may write

$$
G=G_{\Psi} \quad \text { or } G(\Psi), \text { or } \operatorname{Gr}(\Psi) .
$$

The letter $G$ also suggests Gauss, as we now see.

Special Case. Let $\operatorname{dim} X=1$, and let $z$ be a complex coordinate.

Then the Griffiths function is given by

$$
G_{\Psi}(z)=\frac{1}{\rho} \frac{\partial^{2} \log \rho}{\partial z \partial \bar{z}} .
$$

Proof. This follows immediately from the definition.

The function $-G$ is called the Gauss curvature. Note that when $\operatorname{dim} X=1$, then

$$
G=\operatorname{Ric}(\Psi) / \Psi .
$$

Classically, up to Chern and Kobayashi, the Gauss curvature is used. Griffiths started using a notation in which the minus sign is systematically obliterated (see his footnote in [Gri]), and I strongly approve.

Example 3.1. Let $X=\mathbf{D}_{a}$ be the disc of radius $a$ with the volume form

$$
\Psi_{a}=\frac{2 a^{2}}{\left(a^{2}-|z|^{2}\right)^{2}} \frac{\sqrt{-1}}{2 \pi} d z \wedge d \bar{z}
$$

Then

$$
\operatorname{Ric}\left(\Psi_{a}\right)=\Psi_{a} \text { and so } G\left(\Psi_{a}\right)=1 \text {. }
$$

Proof. Immediate from the definitions.

In classical terms, the Gauss curvature of the disc is -1 . We put the factor $2 a^{2}$ in the definition of $\Psi_{a}$ so that we would come out with $G=1$ and Gauss curvature -1 for the hyperbolic disc. We may call $\Psi_{a}$ the normalized hyperbolic form on the disc $\mathbf{D}_{a}$.

Example 3.2. Similarly on the polydisc, let $a=\left(a_{1}, \ldots, a_{n}\right)$ be a sequence of positive numbers;

$$
\mathbf{D}_{a}^{n}=\mathbf{D}^{n}(a)=\mathbf{D}_{a_{1}} \times \cdots \times \mathbf{D}_{a_{n}}
$$


We let $\Psi_{a}^{(n)}$ be the product of the normalized hyperbolic forms on each factor, so that

$$
\Psi_{a}^{(n)}\left(z_{1}, \ldots, z_{n}\right)=\prod_{j=1}^{n} \Psi_{a}\left(z_{j}\right),
$$

where the product is of course the alternating product. Again we call $\Psi_{a}^{(n)}$ the normalized hyperbolic volume form on the polydisc. For this volume form we have

$$
\frac{1}{n !} \operatorname{Ric}\left(\Psi_{a}^{(n)}\right)^{n}=\Psi_{a}^{(n)} \quad \text { so } \quad G\left(\Psi_{a}^{(n)}\right)=1
$$

Indeed, the variables separate, and

$$
\operatorname{Ric}\left(\Psi_{a}^{(n)}\right)\left(z_{1}, \ldots, z_{n}\right)=\sum_{j} \operatorname{Ric} \Psi_{a_{j}}\left(z_{j}\right) .
$$

Taking the $n$th power and using Example 3.1 in one variable, we get the desired relationship.

Unless otherwise specified, all the numbers $a_{1}, \ldots, a_{n}$ will be assumed equal to the same number $a$, in which case we also write $\Psi_{a}^{(n)}$ for the volume form.

Example 3.3. In the case when $G(\Psi)$ is constant and the Ricci form is positive, the manifold is called Einsteinian. A positive constant multiple of the Ricci form is then taken as defining a hermitian metric, which is called an Einstein-Kähler metric.

Functoriality 3.4. Let $X, Y$ have the same dimension. Let $f: Y \rightarrow X$ be a holomorphic mapping. Let $\Psi_{X}$ be a volume form on $X$. Then

$$
\operatorname{Ric}\left(f^{*} \Psi_{X}\right)=f^{*} \operatorname{Ric}\left(\Psi_{X}\right)
$$

wherever $f^{*} \Psi_{X}$ is positive.

If $\Psi_{Y}$ is a volume form on $Y$ then there is a function $u \geqq 0$ such that $f^{*} \Psi_{X}=u \Psi_{Y}$, and we have

$$
\operatorname{Ric}\left(f^{*} \Psi_{X}\right)=\operatorname{Ric}\left(\Psi_{Y}\right)+d d^{c} \log u \text { wherever } u \neq 0 .
$$

Both assertions are immediate from the definitions.

Maximum Principle 3.5. Let $u$ be a real function $>0$ on $Y$. Let $y_{0} \in Y$ be a point such that $u\left(y_{0}\right)$ is a maximum. Then

$$
\left(d d^{c} \log u\right)\left(y_{0}\right) \leqq 0 .
$$

Proof. Given a complex tangent vector $v$ in the complex tangent space at $y_{0}$ there exists an imbedding $f: U \rightarrow Y$ of an open disc $U$ centered at 0 in $\mathbf{C}$ and a tangent vector (complex number) $w$ such that $f(0)=y_{0}$ and such that $d f(0) w=v$. By pull-back, it suffices to prove the negativity of the form $d d^{c} \log (u \circ f)$ at 0 . Hence without loss of generality, we may assume $X=U$. With respect to a complex coordinate $z=x+i y, d d^{c} \log u$ is represented on $U$ by

$$
\left(\frac{\partial}{\partial x^{2}}+\frac{\partial}{\partial y^{2}}\right)(\log u) \frac{1}{2 \pi} d x \wedge d y,
$$

which is $\leqq 0$ at a maximum for $u$ by elementary calculus. 
Lemma 3.6. Let $\omega_{1}, \ldots, \omega_{n}$ be positive $(1,1)$-forms on the complex manifold $X$ of dimension $n$. Then

$$
\omega_{1} \wedge \cdots \wedge \omega_{n}
$$

is a volume form. Furthermore, if $\omega_{i} \leqq \eta_{i}$ for $i=1, \ldots, n$ then

$$
\omega_{1} \wedge \cdots \wedge \omega_{n} \leqq \eta_{1} \wedge \cdots \wedge \eta_{n} \text {. }
$$

Proof. Left to the reader.

In particular, a positive $(1,1)$-form $\omega$ defines a hermitian metric, and $\omega^{n} / n$ ! is a volume form, said to be associated with the metric, or with $\omega$.

On a compact subset of $X$, two positive forms (volume or $(1,1)$ ) are of the same order of magnitude. That is, given, say, two volume forms $\Psi_{1}$ and $\Psi_{2}$ on $X$, and $K$ a compact set, there exists a number $c>0$ such that

$$
\Psi_{1} \leqq c \Psi_{2} \text { on } K
$$

This will be applied for instance when $X$ itself is compact, and when $\Psi_{1}, \Psi_{2}$ are $\Psi$ and $\operatorname{Ric}(\Psi)^{n}$ in case $\operatorname{Ric}(\Psi)$ is positive.

Remark. In this section, for simplicity of language, we limited ourselves to volume forms in the strict sense, that is "positive" meant "strictly positive". In the next section, we shall relax this condition and discuss pseudo volume forms. In important applications, it turns out that $\Psi \geqq 0$ but $\operatorname{Ric}(\Psi)>0$. Thus it may be useful to consider the inverse of the Griffiths function, namely, when $\operatorname{Ric}(\Psi)$ is positive, to consider

$$
\Psi / \frac{1}{n !} \operatorname{Ric}(\Psi)^{n}
$$

and give this an appropriate name.

\section{\$4. Distance and measure decreasing maps}

We begin with a one-dimensional result in a simple context to see the basic pattern of proof.

Theorem 4.1 (Ahlfors' Lemma). Let $f: \mathbf{D}_{a} \rightarrow X$ be a holomorphic map of the disc of radius a into a Riemann surface (one-dimensional complex manifold). Let $\Psi_{X}$ be a volume form on $X$ and $\Psi_{a}$ the normalized hyperbolic form on $\mathbf{D}_{a}$. Assume that there exists a number $B>0$ such that $G\left(\Psi_{X}\right) \geqq B$. Then

$$
f^{*} \Psi_{X} \leqq(1 / B) \Psi_{a} .
$$

Proof. Write $f^{*} \Psi_{X}=u \Psi_{a}$, with a function $u$. We take two steps.

First step. We reduce to the case when $u$ has a maximum in $\mathbf{D}_{a}$. Let $0<t<a$. Then $\Psi_{t} \rightarrow \Psi_{a}$ as $t \rightarrow a$. Let $u_{t}$ be the function such that

$$
f^{*} \Psi_{X}=u_{t} \Psi_{t} \text { on } \mathbf{D}_{t} \text {. }
$$

Then for each $z \in \mathbf{D}_{a}, u_{t}(z) \rightarrow u(z)$ as $t \rightarrow a$. Write

$$
f^{*} \Psi_{X}=h \frac{\sqrt{-1}}{2 \pi} d z \wedge d \bar{z} \text { on } \mathbf{D}_{a} .
$$


Then $h$ is bounded on $\mathbf{D}_{t}^{\text {cl }}$ and

$$
u_{t}(z)=h(z)\left(t^{2}-|z|^{2}\right) / 2 t^{2},
$$

so $u_{t}(z) \rightarrow 0$ as $|z| \rightarrow t$. Hence $u_{t}$ has a maximum in $\mathbf{D}_{t}$, and it suffices to prove the inequality of the theorem for $u_{t}$, as desired.

Second step. Assume $u\left(z_{0}\right)$ is a maximum for $u$ in $\mathbf{D}_{a}$. If $u\left(z_{0}\right)=0$, we are done, because $u=0$. Suppose $u\left(z_{0}\right) \neq 0$. Then $f$ restricts to a local isomorphism $f: U \rightarrow X$ on a neighborhood $U$ of $z_{0}$. Then by hypothesis,

$$
\Psi_{a}+d d^{c} \log U=f^{*} \operatorname{Ric}\left(\Psi_{X}\right) \geqq B f^{*} \Psi_{X} .
$$

The function $\log u$ has a local maximum at $z_{0}$, and hence

$$
\left(d d^{c} \log u\right)\left(z_{0}\right) \leqq 0
$$

by the maximum principle 3.5 . This proves that

$$
\Psi_{a}\left(z_{0}\right) \geqq B f^{*} \Psi_{X}\left(z_{0}\right),
$$

and therefore $u\left(z_{0}\right) \leqq 1 / B$. Since $u\left(z_{0}\right)$ is a maximum value, this also proves the theorem.

The first higher-dimensional version of the Schwarz-Ahlfors lemma is apparently due to Grauert-Reckziegel [G-R] and Dinghas [Di]. Extensions and clarifications of the differential geometric conditions under which the result is true were then given by Chern [Ch], Griffiths [Gri], Kobayashi [Ko 1, Ko 2], and Reckziegel [Re]. In [G-R], some basic properties are proved for a notion which extends hyperbolicity in one direction, involving "length functions" (called "differentialmetrik" in [G-R]), which will be briefly mentioned in $\$ 7$. Such length functions have the advantage of applying also to singular analytic spaces as shown in Reckziegel's thesis [Re]. To keep the language simpler so that we can use the better-known formalism of differential forms, I shall stick to the case of manifolds. For instance, I reproduce the following two theorems as in Kobayashi. However, the analogues occur in [G-R] and [Re], who work with a differential geometric definition of hyperbolicity, in the more general context of length functions.

Theorem 4.2. Let $X$ be a complex manifold with hermitian structure defined by a positive $(1,1)$-form $\omega_{X}$. Assume that there is a constant $c$ such that for every holomorphic map $f: \mathbf{D} \rightarrow X$ we have

$$
f^{*} \omega_{X} \leqq c \Psi_{1}
$$

Then $X$ is hyperbolic.

Proof. After multiplying $\omega_{X}$ by a suitable constant, we may assume that $c=1$. Then $f$ is distance decreasing, and in particular the Kobayashi distance is bounded from below by the hermitian distance, which is non-trivial, so the manifold is hyperbolic. 
The above theorem is then applied to the following situation.

Theorem 4.3. Let $X$ be a complex hermitian manifold, with hermitian metric associated with the positive $(1,1)$-form $\omega_{X}$. For each one-dimensional immersed submanifold $Y$ of $X$, let $\omega_{Y}=\omega_{X} \mid Y$, so $\omega_{Y}$ is a volume form on $Y$. Assume that there is a number $B>0$ such that $G\left(\omega_{Y}\right) \geqq B$ for all such $Y$. Let

$$
f: \mathbf{D}_{a} \rightarrow X
$$

be a holomorphic map. Then

$$
f^{*} \omega_{X} \leqq(1 / B) \Psi_{a},
$$

and $X$ is hyperbolic.

Proof. Again we reduce the statement to the case when $f^{*} \omega_{X}=u \Psi_{a}$ and $u$ has a maximum $u\left(z_{0}\right)$ in $\mathbf{D}_{a}$. If $u\left(z_{0}\right)=0$, we are done. If $u\left(z_{0}\right) \neq 0$ then $f$ is a local isomorphism at $z_{0}$, and $f: U \rightarrow f(U)=Y$ thus gives an isomorphism of a neighborhood of $z_{0}$ with a one-dimensional complex submanifold of $X$. We can now apply the hypothesis and Theorem 4.1 to complete the proof of the first statement. That $X$ is hyperbolic follows by applying the criterion of Theorem 4.2.

In [Ko 2], Chapter IX, Problem 5, Kobayashi raises the problem whether the converse is true. We phrase this here as

Problem 4.4. Let $X$ be a non-singular hyperbolic variety. Does there exist a positive $(1,1)$-form $\omega$ and a number $B>0$ such that for every one-dimensional immersed submanifold $Y$ in $X$, we have

$$
G_{\omega \mid Y} \geqq B .
$$

This problem involving a $(1,1)$-form (condition 4 in the introduction) is the stronger version of an alternative converse to Theorem 4.3, in which this $(1,1)$-form is replaced by a length function, originally introduced in different contexts of hyperbolicity by Grauert-Reckziegel [G-R] and Kobayashi [Ko 4]. We shall discuss this notion and an application in $\$ 7$. For a one-dimensional complex manifold the notion of length function coincides with that of a hermitian metric, and the point is that $Y$ in Problem 4.4 is one-dimensional. Thus an intermediate problem would be to show that if $X$ is hyperbolic, then there exists a length function whose Griffiths function satisfies the desired inequality. Recall here that by our conventions, a variety is projective, so compact.

Next we have a result in the equidimensional case which evolved from Dinghas [Di] and Chern [Ch] to Kobayashi [Ko 1], see also Griffiths [Gri] and Kobayashi-Ochiai [K-O]. The proof will repeat the pattern of the proof of Ahlfors' Lemma.

Theorem 4.5. Let $X$ be a compact complex manifold. Let $\Psi_{X}$ be a volume form such that $\operatorname{Ric}\left(\Psi_{X}\right)$ is positive. Let $\operatorname{dim} X=n$. Let $\Psi_{a}^{(n)}$ be the normalized hyperbolic volume form on $\mathbf{D}_{a}^{n}$. Then there exists a constant $c>0$ such that for all holomorphic maps

$$
f: \mathbf{D}_{a}^{n} \rightarrow X
$$


we have

$$
f^{*} \Psi_{X} \leqq c \Psi_{a}^{(n)}
$$

Proof. Let $0<t<a$. By reproducing exactly the argument in Step 1 of Theorem 4.1 for the volume forms, we reduce the proof to the case when

$$
f^{*} \Psi_{X}=u \Psi_{a}^{(n)}
$$

and $u \geqq 0$ has a maximum at $z_{0}$ in $\mathbf{D}_{a}^{n}$. Again if this maximum is 0 , we are done, so we may assume the maximum $\neq 0$. Then

$$
d d^{c} \log u=f^{*} \operatorname{Ric}\left(\Psi_{X}\right)-\operatorname{Ric}\left(\Psi_{a}^{(n)}\right)
$$

wherever $u \neq 0$, and at the maximum point we know by the maximum principle 3.5 that the left side is $\leqq 0$. Hence we find

$$
f^{*} \operatorname{Ric}\left(\Psi_{X}\right)\left(z_{0}\right) \leqq \operatorname{Ric}\left(\Psi_{a}^{(n)}\right)\left(z_{0}\right) .
$$

We take the $n$th power of each side. The divided $n$th power $(1 / n !) \operatorname{Ric}\left(\Psi_{a}^{(n)}\right)^{n}$ is just $\Psi_{a}^{(n)}$, and since we assumed $\operatorname{Ric}\left(\Psi_{X}\right)$ positive, we have trivially

$$
\Psi_{X} \leqq c \operatorname{Ric}\left(\Psi_{X}\right)^{n} \quad \text { for some constant } c>0 .
$$

Hence

$$
f^{*} \Psi_{X}\left(z_{0}\right) \ll f^{*} \operatorname{Ric}\left(\Psi_{X}\right)^{n}\left(z_{0}\right) \ll \Psi_{a}^{(n)}\left(z_{0}\right),
$$

where the sign $\ll$ means "less than a constant times". The constant depends only on the given form $\Psi_{X}$ and $X$, but is independent of $f$. This shows that $u\left(z_{0}\right)$ is bounded by such a constant, and since $u\left(z_{0}\right)$ is a maximum for $u$, this proves the theorem.

Remarks. As pointed out by Kobayashi in his book [Ko 2], the statements generalize when the polydiscs are replaced by various bounded domains. What we needed was a starlike property, to use the trick with $0<t<a$, and the fact that the hyperbolic metric goes to infinity toward the boundary. Then the same arguments go through. This is important for applications to bounded (symmetric) domains.

The statement will be extended to pseudo canonical varieties in the next section.

Also, the same proof and conclusion hold in the non-compact case, provided there exists an inequality $\Psi_{X} \ll \operatorname{Ric}\left(\Psi_{X}\right)^{n}$. For applications in the next section, we give the appropriate formulation of the result under more general conditions.

Let $X$ be a complex manifold of dimension $n$. By a pseudo volume form $\Psi$ or $\Psi_{X}$ we shall mean a continuous $(n, n)$-form which is $C^{\infty}$ outside a proper analytic subset, and which locally in terms of complex coordinates can be expressed as

$$
\Psi(z)=|g(z)|^{2 q} h(z) \Phi(z)
$$


where

$q$ is some fixed rational number $>0$;

$g$ is holomorphic not identically zero;

$h$ is $C^{\infty}$ and $>0$;

and

$$
\Phi(z)=\prod_{i=1}^{n} \frac{\sqrt{-1}}{2 \pi} d z_{i} \wedge d \bar{z}_{i} .
$$

The definition is a variation of other possible definitions which weaken the conditions of a volume form, and is adjusted for the applications we have in mind here. Zeros are allowed on a proper analytic set.

We can define $\operatorname{Ric}(\Psi)$ for a pseudo volume form just as we did for a volume form, by the formula

$$
\operatorname{Ric}(\Psi)=d d^{c} \log h .
$$

Since $g$ is assumed holomorphic, $d d^{c} \log |g|^{2 q}=0$ wherever $g \neq 0$.

Theorem 4.5'. Let $X$ be a complex manifold. Let $\Psi_{X}$ be a pseudo volume form such that $\operatorname{Ric}\left(\Psi_{X}\right)$ is positive and such that there exists a constant $c^{\prime}>0$ for which

$$
\Psi_{X} \leqq c^{\prime} \operatorname{Ric}\left(\Psi_{X}\right)^{n}
$$

(Such a $c^{\prime}$ exists if $X$ is compact.) Then there exists a constant $c>0$ such that for all holomorphic maps

$$
f: \mathbf{D}_{a}^{n} \rightarrow X
$$

we have

$$
f^{*} \Psi_{X} \leqq c \Psi_{a}^{(n)} .
$$

The proof is identical with the previous proof. The arguments are valid under the weaker assumptions.

We now pass to measure theoretic considerations.

Let $X$ be a complex manifold, with volume or pseudo volume form $\Psi$. Then $\Psi$ defines a positive functional on $C_{c}(X)$ (continuous functions with compact support) by

$$
\varphi \mapsto \int_{X} \varphi \Psi
$$

Hence there is a unique regular positive measure $\mu_{\Psi}$ such that for all $\varphi \in C_{c}(X)$ we have

$$
\int_{X} \varphi \Psi=\int_{X} \varphi d \mu_{\Psi}
$$

Let $Z, X$ be complex analytic spaces. We take for granted that if $f: Z \rightarrow X$ is analytic, and $U$ open in $Z$, then $f(U)$ is Borel measurable in $X$, in fact equal to a countable union of analytic subspaces of $X$. We shall assume that there is a countable sequence of analytic maps $\left\{f_{i}\right\}$ such that $\bigcup f_{i}(Z)$ covers $X$. We let 
$\mu_{Z}$ be a positive regular Borel measure on $Z$. In the application, we shall take

$$
\begin{aligned}
& Z=\mathbf{D}_{1}^{n}=n \text {-fold product of the unit disc; } \\
& \mu_{Z}=\text { hyperbolic metric } \Psi_{1}^{(n)} .
\end{aligned}
$$

Let $B$ be measurable in $X$, and consider sequences $f_{i}: Z \rightarrow X$ of analytic maps, and open sets $U_{i}$ in $Z$ such that

$$
B \subset \bigcup f_{i}\left(U_{i}\right)
$$

We define the Kobayashi measure (with respect to $\mu_{Z}$ )

$$
\mu_{\mathrm{Kob}, X}(B)=\inf \sum_{i=1}^{\infty} \mu_{Z}\left(U_{i}\right)
$$

where the inf is taken over all sequences $\left\{f_{i}\right\}$ and sets $\left\{U_{i}\right\}$ prescribed above. It is an exercise in basic techniques of measure theory to show that $\mu_{\mathrm{Kob}, X}$ is a measure.

Since the open sets generate the $\sigma$-algebra of Borel measurable sets, it follows that if $A$ is measurable in $Z$ and $f$ is holomorphic, then $f(A)$ is measurable. Furthermore, a regular measure satisfies the property that the measure of a set is the inf of the measures of the open sets containing it. Hence in the definition of the Kobayashi measure, instead of taking open sets $U_{i}$ we could take measurable sets $A_{i}$ in $Z$.

Let $Y, X$ be complex analytic spaces, and let $f: Y \rightarrow X$ be an analytic map. Let $\mu_{X}, \mu_{Y}$ be regular Borel measures on $X$ and $Y$. We say that $f$ is measure decreasing if for every open set $U$ in $Y$ we have

$$
\mu_{X}(f(U)) \leqq \mu_{Y}(U)
$$

or equivalently, for every measureable set $A$ in $Y$ we have

$$
\mu_{X}(f(A)) \leqq \mu_{Y}(A) .
$$

Example. Let $X, Y$ be complex manifolds with $\operatorname{dim} X=\operatorname{dim} Y$. Let $f: Y \rightarrow X$ be holomorphic. Let $\Psi_{X}, \Psi_{Y}$ be volume or pseudo volume forms on $X$ and $Y$ respectively, defining measures $\mu_{X}, \mu_{Y}$. Assume that

$$
f^{*} \Psi_{X} \leqq \Psi_{Y}
$$

Then $f$ is measure decreasing. Indeed, the set of points $y \in Y$ such that $d f(y)$ is singular is an analytic subset $S$, and $f(S)$ has measure 0 . On the open complement of $S$, one sees at once that $f$ is measure decreasing, so $f$ is measure decreasing. This can be applied to the cases of Theorems 4.5 and 4.5', after multiplying $\Psi_{X}$ with a sufficiently small positive constant.

From now on, we let $Z=\mathbf{D}_{1}^{n}$ with the normalized hyperbolic measure $\mu_{1}^{(n)}$ corresponding to the volume form $\Psi_{1}^{(n)}$. For every complex manifold $X$ we then obtain the Kobayashi measure $\mu_{\mathrm{Kob}, X}$. 
The following properties are immediate, concerning measure decreasing maps.

MD 1. Let $f: Y \rightarrow X$ be holomorphic, and $\operatorname{dim} Y=\operatorname{dim} X=n$. Then $f$ is measure decreasing for the Kobayashi measures.

MD 2. Let $\nu$ be a measure on $X$ such that every holomorphic map $f: \mathbf{D}_{1}^{n} \rightarrow X$ is measure decreasing from $\mu_{1}^{(n)}$ to $\nu$. Then $\nu \leqq \mu_{\mathrm{Kob}, X}$.

MD 3. If all holomorphic maps $f: \mathbf{D}_{1}^{n} \rightarrow X$ are measure decreasing, and if $\mu_{X}(V)>0$ for all open sets $V$ in $X$, then $\mu_{\mathrm{Kob}, X}(V)>0$ for all open $V$ in $X$.

We define $X$ to be measure hyperbolic if $\mu_{\mathrm{Kob}, X}(V)>0$ for all non-empty open sets $V$ in $X$.

Corollary 4.6. If $X$ is a compact manifold and $\operatorname{Ric}\left(\Psi_{X}\right)$ is positive then $X$ is measure hyperbolic.

Proof. After multiplying $\Psi_{X}$ by a sufficiently small constant, we see from Theorem 4.5 that holomorphic maps as above are measure decreasing, and hence that $X$ is measure hyperbolic.

In order to avoid mentioning constants, one could define a map to be essentially volume decreasing by allowing a positive constant in comparing the volumes.

We also formulate the corollary for pseudo volume forms.

Corollary 4.6'. Let $X$ be a complex manifold, and let $\Psi_{X}$ be a pseudo volume form. Assume that $\operatorname{Ric}\left(\Psi_{X}\right)$ is positive, and that there exists $c^{\prime}>0$ such that

$$
\Psi_{X} \leqq c^{\prime} \operatorname{Ric}\left(\Psi_{X}\right)^{n}
$$

(If $X$ is compact, then $c^{\prime}$ always exists.) Then $X$ is measure hyperbolic.

For our purposes here, I quote without proof Theorem 1.10 of Kobayashi's Chapter IX:

Theorem 4.7. Let $X$ be a complex manifold which is hyperbolic. Then $X$ is measure hyperbolic.

Then I make the conjecture

Conjecture 4.8. Let $X$ be a variety. Then $X$ is hyperbolic if and only if all subvarieties of $X$ (including $X$ itself) are measure hyperbolic.

The implication in one direction comes from Kobayashi's theorem (which is presumably valid also for varieties, even singular, with the same proof he gives in his book), and from Brody's characterization of hyperbolic varieties by the condition that they admit no complex lines (non-constant). This condition also applies to subvarieties. Thus the content of the conjecture lies in the converse: if all subvarieties are measure hyperbolic, then $X$ is hyperbolic. 
In the next section, we look at the algebraic geometric context for this conjecture, and its relation with another conjecture of Kobayashi.

In connection with algebraic geometry, it is unknown if the property of being measure hyperbolic is birationally invariant. To deal with this problem, Yau [Ya] uses meromorphic maps instead of holomorphic maps to define a variant of the Kobayashi measure. He then has to prove the functorial properties concerning pull-backs of forms, but once this is done, his definition makes the birational invariance obvious.

\section{§5. Pseudo canonical varieties (general type)}

With the simultaneous appearance of the papers by Griffiths [Gri] and Kobayashi-Ochiai [K-O], it was realized that arguments which applied to compact manifolds with positive Ricci form, combined with results of Kodaira, also apply to varieties where this condition is weakened, and which classically are called "of general type". Roughly speaking, given a certain property, the weakening of this property obtained by requiring that it holds only outside a proper algebraic subset may be called its pseudofication. The purpose of this section is to carry out the arguments concerning this more general case. The above papers are very similar. I follow especially [K-O 1].

To begin, recall a theorem of Kodaira which says that if a compact complex manifold $X$ has a metrized line bundle with positive Chern form then $X$ admits a projective imbedding, cf. Griffiths-Harris, Chapter II, $\S 4$. Let $L$ be a line bundle on $X$. We abbreviate the tensor product $L^{\otimes m}$ by $L^{m}$. We say that $L$ is ample if there exists some $m$ such that a basis of sections $\left(s_{0}, \ldots, s_{N}\right)$ of $H^{0}\left(X, L^{m}\right)$ generates $L^{m}$ at every point and give a projective imbedding

$$
\varphi_{m}=\left(s_{0}, \ldots, s_{N}\right): X \rightarrow \mathbf{P}^{N}
$$

We say that $L$ is very ample if we can take $m_{0}=1$ in the above condition, so already sections of $H^{0}(X, L)$ give the imbedding.

Even if we do not get a projective imbedding by means of a basis for the sections, we still get a rational map into $\mathbf{P}^{N}$. We let

$$
m \underset{\operatorname{div}}{\rightarrow} \infty
$$

denote the property that $m$ tends to infinity, ordered by divisibility. We say that $L$ is pseudo ample if $\varphi_{m}$ is birational, that is $\varphi_{m}$ gives a projective imbedding of a non-empty Zariski open subset, for $m$ large ordered by divisibility.

Suppose $X$ non-singular, and let $K_{X}$ be the canonical bundle. Classically, $X$ has been called canonical if $K_{X}$ is very ample [Gri]. But for the same reason that Grothendieck changed the meaning of "ample" to what it is now, it seems more fruitful to say that $X$ is canonical if $K_{X}$ is ample, and very canonical if $K_{X}$ is very ample. On the other hand, if $K_{X}$ is pseudo ample, then $X$ is usually said to be of general type, but with the support of Griffiths, I shall say that $X$ is 
pseudo canonical to make the terminology functorial with respect to the ideas. Finally, if $X$ is singular, we say that $X$ is of general type or pseudo canonical if some desingularization has this property. We recall a basic elementary fact.

If $X, Y$ are non-singular, then a birational map $X \rightarrow Y$ induces an isomorphism

$$
H^{0}\left(X, K_{X}^{m}\right) \stackrel{\approx}{\rightarrow} H^{0}\left(Y, K_{Y}^{m}\right)
$$

for every positive integer $m$.

Proof. See Hartshorne, Algebraic Geometry, Chapter II, Theorem 8.19. The proof given there when $m=1$ works in general. The idea is that a section of $K_{X}^{m}$ can be represented globally birationally-for instance, as

$$
g\left(d g_{1} \wedge \cdots \wedge d g_{n}\right)^{m},
$$

where $g, g_{1}, \ldots, g_{n}$ are rational functions. If $g_{1}, \ldots, g_{n}$ are local parameters at a point, then $g$ is in the local ring at that point. One now uses the fact that if a rational function is not in the local ring of a point, then it has a divisorial pole passing through that point. Such a pole induces a point on any complete model $X$ or $Y$. This shows that if a rational form as above gives rise to a section of $K_{X}^{m}$ for one model, then it must give rise to a section of $K_{Y}^{m}$ for any other model.

We shall be interested in the dimension

$$
h^{0}\left(X, L^{m}\right)=\operatorname{dim} H^{0}\left(X, L^{m}\right)
$$

for various line bundles $L$, starting with the canonical bundle, but involving other bundles as in $\S 7$. In speaking of estimates, we use the standard notation of number theorists (Vinogradov)

$$
A(m) \ll B(m) \text { for } m \rightarrow \infty
$$

to mean that there is a constant $c$ such that $A(m) \leqq c B(m)$ for all $m$ sufficiently large. If the going to infinity is by divisibility ordering, then sufficiently large is according to this ordering. Following [K-O 1] and its addendum using Kodaira's technique, we shall now construct a pseudo volume form on a non-singular pseudo canonical variety, with positive Ricci form. We recall two lemmas from algebraic geometry.

Lemma 5.1. Let $X$ be a variety of dimension $n$. Let $D$ be a divisor on $X$. Then

$$
h^{0}(m D)=\operatorname{dim} H^{0}(X, m D) \ll m^{n} \quad \text { for } m \rightarrow \infty .
$$

Proof. Let $E$ be a divisor which is ample and such that $D+E$ is ample. Then we have an inclusion

$$
H^{0}(m D) \subset H^{0}(m D+m E) \text {, so } h^{0}(m D) \leqq h^{0}(m D+m E) .
$$

Furthermore, if $E^{\prime}=D+E$ is ample, then

$$
h^{0}\left(m E^{\prime}\right)=\chi\left(m E^{\prime}\right) \text { for } m \text { large }
$$


because the higher cohomology groups vanish for $m$ large, and the Euler characteristic is a polynomial in $m$ of degree $\leqq n$, thus proving the lemma.

Lemma 5.2. Let $X$ be a non-singular variety of dimension $n$. Let $E$ be very ample on $X$, and let $D$ be a divisor on $X$ such that

$$
h^{0}(m D) \gg m^{n} \text { for } m \underset{\operatorname{div}}{\rightarrow} \infty .
$$

Then

$$
h^{0}(m D-E) \gg m^{n} \text { for } m \underset{\mathrm{div}}{\rightarrow} \infty,
$$

in particular, $m D-E$ is linearly equivalent to an effective divisor.

Proof. Without loss of generality, we may replace $E$ by any divisor in its class, and thus we may assume that $E$ is an irreducible non-singular subvariety of $X$ (a hyperplane section, in fact). We have the exact sequence

$$
0 \rightarrow \mathcal{O}(m D-E) \rightarrow \mathcal{O}(m D) \rightarrow \mathcal{O}(m D) \mid E \rightarrow 0,
$$

whence the exact cohomology sequence

$$
\left.0 \rightarrow H^{0}(X, m D-E) \rightarrow H^{0}(X, m D) \rightarrow H^{0}(E, \mathcal{O}(D) \mid E)^{\otimes m}\right)
$$

noting that $\mathcal{O}(m D) \mid E=(\mathcal{O}(D) \mid E)^{\otimes m}$. Applying the first lemma to this invertible sheaf on $E$ we conclude that the dimension of the term on the right is $\ll m^{n-1}$, so $h^{0}(X, m D-E) \gg m^{n}$ for $m$ large, and in particular is positive for $m$ large, whence the lemma follows.

Suppose that $X$ is pseudo canonical. Then $h^{0}\left(X, K_{X}^{m}\right) \gg m^{n}$ for $m$ large, so we can apply the lemma. Let $L$ be a very ample line bundle on $X$. We shall obtain a projective imbedding of $X$ by means of some of the sections in $H^{0}\left(X, K_{X}^{m}\right)$. By Lemma 5.2, for $m$ large there exists a non-trivial holomorphic section $\alpha$ of $K_{X}^{m} \otimes L^{-1}$. Let $\left\{s_{0}, \ldots, s_{N}\right\}$ be a basis of $H^{0}(X, L)$. Then

$$
\alpha \otimes s_{0}, \ldots, \alpha \otimes s_{N}
$$

are linearly independent sections of $H^{0}\left(X, K_{X}^{m}\right)$. Since $\left(s_{0}, \ldots, s_{N}\right)$ gives a projective imbedding of $X$ into $\mathbf{P}^{N}$ because $L$ is assumed very ample, it follows that $\alpha \otimes s_{0}, \ldots, \alpha \otimes s_{N}$ vanish simultaneously only at the zeros of $\alpha$, but nevertheless give the same projective imbedding, which is determined only by their ratios. Then

$$
\alpha \bar{\alpha} \otimes \sum s_{j} \otimes \bar{s}_{j}
$$

may be considered as a section of

$$
\left(K_{X}^{m} L^{-1}\right) L \otimes\left(\bar{K}_{X}^{m} \bar{L}^{-1}\right) \bar{L}=K_{X}^{m} \otimes \bar{K}_{X}^{m}
$$

and can be locally expressed in terms of complex coordinates in the form

$$
|g(z)|^{2} \sum_{j=1}^{n}\left|g_{j}(z)\right|^{2} \Phi(z)^{\otimes m},
$$

where as usual $\Phi(z)$ is the standard euclidean volume form on $\mathbf{C}^{n}$, while $g(z)$, $g_{0}(z), \ldots, g_{N}(z)$ are local holomorphic functions representing $\alpha, s_{0}, \ldots, s_{N}$ respectively. 
Let

$$
h(z)=\left(\sum_{j=1}^{n}\left|g_{j}(z)\right|^{2}\right)^{1 / m}
$$

Then there is a unique pseudo volume form $\Psi$ on $X$ which has the local expression

$$
\Psi(z)=|g(z)|^{2 / m} h(z) \Phi(z) .
$$

Furthermore $\operatorname{Ric}(\Psi)$ is positive, because $\operatorname{Ric}(\Psi)$ is the pull-back of the Fubini-Study form on $\mathbf{P}^{N}$ by the projective imbedding.

In particular, we have proved:

Theorem 5.3 (Kodaira, Kobayashi-Ochiai). Let $X$ be a non-singular pseudo canonical variety. Then $X$ admits a pseudo volume form $\Psi_{X}$ with $\operatorname{Ric}\left(\Psi_{X}\right)$ positive, and $X$ is measure hyperbolic.

As to the converse, we have on the one hand:

Conjecture 5.4 (Kobayashi, see [Ko 2, Chapter IX]). If $X$ is measure hyperbolic, then $X$ is pseudo canonical.

This would give the neat statement that

A non-singular variety is pseudo canonical if and only if it is measure hyperbolic.

Kobayashi's conjecture is known for surfaces, through the paper of GreenGriffiths [Gr-Gr], completed in one remaining case (arising from the classification of surfaces) by Bogomolov and Mumford. Cf. the appendix of Mori-Mukai [Mo-Mu].

On the other hand, the converse in the differential geometric context is also a problem, raised by Kobayashi [Ko 3, Theorem 7.1 and p. 377], namely:

If $X$ is non-singular, and there exists a pseudo volume form with positive Ricci form, is $X$ pseudo canonical?

This would be a pseudofication of Kodaira's imbedding theorem.

Conjecture 5.5 (Green-Griffiths). Let $X$ be a pseudo canonical variety. Let $f: \mathbf{C} \rightarrow X$ be holomorphic non-constant. Then the image of $f$ is contained in a proper subvariety.

For more precise information on this, see $\S 8$.

I would conjecture:

Conjecture 5.6. $A$ variety $X$ is hyperbolic if and only if every subvariety (including $X$ itself) is pseudo canonical.

This would give an algebraic condition characterizing hyperbolicity on algebraic varieties. If $X$ is hyperbolic then $X$ is measure hyperbolic by Kobayashi's Theorem 4.7, and if $X$ is a surface then $X$ is pseudo canonical as mentioned above (in general it is Conjecture 5.4). Conversely, if $X$ is pseudo canonical, then the Green-Griffiths conjecture applies, and if all subvarieties are assumed pseudo canonical, then by that conjecture $X$ is hyperbolic; but the question remains even on surfaces. 
Conjecture 5.6 is consistent with known results about the following special example. Let $X$ be a subvariety of an abelian variety, which does not contain the translate of a subtorus of dimension $>0$. Then by Theorems 1 and $1^{\prime}$ of [Ya] (using fundamental results of litaka), it follows that $X$ is pseudo canonical. In particular, every subvariety of $X$ is also pseudo canonical, and we already know by Green's Theorem 2.4 that $X$ is hyperbolic.

We now come to the diophantine connection.

Conjecture 5.7. Let $X$ be a pseudo canonical variety. Then the rational points in every finitely generated field $F$ are not Zariski dense.

The history of this conjecture will be recalled in an appendix. Here it is relevant to note that Noguchi proved the analogue of this conjecture for algebraic families, under the hypothesis that the cotangent bundle is ample. A vector bundle $E$ is defined to be ample if the tautological line bundle on the associated projective bundle $\mathbf{P E}$ is ample, or equivalently, if $\mathscr{E}$ is the sheaf of sections, then for any coherent sheaf $\mathscr{F}, \mathscr{F} \otimes S^{m} \mathscr{E}$ is generated by its global sections for $m$ sufficiently large. For an excellent foundational discussion of ample vector bundles, see Hartshorne [Ha]. Ampleness implies that for $m$ large, a basis for the global sections of $S^{m} E$ give a projective imbedding in the Grassmanian, but in dimension $>1$ the converse is not true. We postpone to the next section a discussion of the condition of ampleness and other conditions, to have an appropriate structural setting for them.

We note that Conjectures 5.6 and 5.7 imply Conjecture 2.3 that a hyperbolic variety is mordellic. Thus Conjecture 5.6 replaces the complex analytic condition by an algebraic one. Vojta, in connection with his translation of Nevanlinna theory into number theory [Vo] conjectures that for a pseudo canonical variety over a number field, the proper algebraic subset which may contain infinitely many rational points can be taken independently of all number fields, which is a refinement of Conjecture 5.7 over number fields. But I conjecture the more precise statement expressed geometrically as follows. Recall that the exceptional $\operatorname{set} \operatorname{Exc}(X)$ is the Zariski closure of all images of non-constant holomorphic maps of $\mathbf{C}$ into $X$.

Conjecture 5.8. Let $X$ be pseudo canonical. Then $\operatorname{Exc}(X) \neq X$ and the complement of $\operatorname{Exc}(X)$ is mordellic.

We shall describe one possible approach to the exceptional set in $\S 8$.

Another approach may come from Theorem 2 of [Ax] already mentioned in the proof of Theorem 2.6. Transposed to the present case, Ax's technique may show how holomorphic curves yield integrable submanifolds, giving insight into the Zariski closure as in Ax's theorem.

We observe that there has been a systematic pattern of conditions which admit a weakening, like volume forms or ampleness which weaken to pseudo volume forms and pseudo ampleness. Recall that we define a variety $X$ to be pseudo hyperbolic if the exceptional set is a proper subset. Similarly, we define $X$ to be pseudo mordellic if there exists a proper algebraic subset $Y$ such that $X-Y$ is mordellic. Then by Conjecture 5.8, pseudo canonical implies pseudo 
hyperbolic, and also implies pseudo mordellic. Furthermore, we can take $Y=\operatorname{Exc}(X)$. The question arises under what conditions does pseudo mordellic imply pseudo canonical, and which varieties are pseudo mordellic without being pseudo canonical, or which are mordellic without being hyperbolic. Are there any? My guess is no.

Conjecture 5.9. If $X$ is pseudo mordellic then $X$ is pseudo canonical.

The modular varieties are pseudo canonical in high dimension. I am told by experts that the structure of the exceptional set and of the boundary components after desingularization is mostly a mystery. The literature on moduli varieties is open ended, so I just mention two papers by Harris-Mumford [Ha-M] and Tai [Ta] proving the pseudo ampleness. It should be emphasized that although the open part of the moduli space which is a quotient of a bounded domain is hyperbolic (both in the Brody and the Kobayashi sense), nevertheless in general, its compactification is not. There is a very strong tendency for this compactification to contain rational curves, or non-constant holomorphic images of $\mathbf{C}$, which must therefore intersect the boundary at infinity.

Finally we observe that the property in Conjecture 5.7 is definitely weaker than the property in Conjecture 5.8. For example, let $X=\mathbf{P}^{1} \times C$, where $C$ is a curve of genus 2 , defined over a number field. Then for every number field $F, X(F)$ is not Zariski dense in $X$ by Falting's theorem, but $X$ is not pseudo canonical. This phenomenon has to do with the canonical class being somewhat less than pseudo ample, e.g. having less than maximal Kodaira dimension.

At this point, we have stated and discussed one by one the problems and theorems summarized in the introduction. If (as I hope) all the conjectures made there are true, then they give a very clear picture of the relations between the diophantine property of having finitely or infinitely many rational points, and the other properties coming from algebraic geometry, differential geometry, and measure theory, because of the necessary and sufficient conditions in all possible directions, except possibly for $3 \Rightarrow 4$.

Warning. We have applied the property of being mordellic to an open algebraic subset of a variety, when discussing pseudo canonical and pseudo mordellic varieties. We remind the reader that for open subsets of varieties, hyperbolic is not equivalent to mordellic, as one sees from Theorem 2.6. Green has also constructed an algebraic example of a Zariski open set which is Brody hyperbolic but not Kobayashi hyperbolic. The "hyperbolicity" of affine varieties has to do with the finiteness of "integral points".

\section{§6. Minimal models}

In this section, we mention still another connection. The reader interested in pursuing the previous ideas can skip immediately to the next section.

The property of being pseudo canonical allows for exceptional subvarieties on a variety, which may have infinitely many rational points. One tries to get rid of these by blowing them down, and thus we can also consider hyperbolicity in the following context. We say that a variety is absolutely minimal if every 
birational map $f: Y \rightarrow X$ with $Y$ non-singular is a morphism. I reproduce here part of Theorem 2.1 of [Ko 2, Chapter VIII].

Theorem 6.1. Every hyperbolic variety is absolutely minimal.

Under what conditions is the converse true for pseudo canonical varieties? I once hoped that a minimal model would be hyperbolic in order to show that Conjecture 2.3 implies 5.8, but that it is not true in general. Artin pointed out to me that one can construct examples (more or less generically) as follows. We consider a hypersurface of degree 5 in $\mathbf{P}^{3}$, defined by the general polynomial equation

$$
P\left(x_{0}, \ldots, x_{3}\right)=0
$$

We impose the condition that it contains a rational curve of degree 5 with simple nodes, say six nodes (to make it have genus 0 ), and adjust coordinates so that the curve is defined by the equation

$$
x_{0}=0 \text {. }
$$

The general solution to this gives a non-singular surface which is absolutely minimal. There cannot be a hyperbolic model of its function field since by Kobayashi's Theorem 5.10 the map from the quintic surface to such a model would be a morphism, and on the other hand, the rational curve cannot be blown down since it is numerically positive.

Concerning the existence of minimal models, it may be useful for the reader to recall here two known results. In the case of surfaces, one has the following theorem of Mumford [Mu].

Let $X$ be a non-singular pseudo canonical surface. Let

$$
R=\bigoplus_{m=0}^{\infty} H^{0}\left(X, K_{X}^{m}\right)
$$

and let $X^{\prime}=\operatorname{Proj}(R)$. Then $X^{\prime}$ is a normal surface with a finite number of rational double points. A minimal desingularization of $X^{\prime}$ exists, and it is absolutely minmal model of $X$.

For other theorems concerning the canonical ring of surfaces, see Bombieri [Bom]. In arbitrary dimensions, we have the following theorem of Kobayashi [Ko 2], Chapter VIII, Theorem 3.6.

Let $X$ be a non-singular variety such that $K_{X}^{m}$ has no base point for some $m$, that is, given $x \in X$ there is a section $s$ of $K_{X}^{m}$ with $s(x) \neq 0$. Then $X$ is relatively minimal.

The recent literature on minimal models and canonical singularities is very extensive, and there is no question of attempting to survey it here. It may help the reader just to refer to Kawamata [Ka 1, Ka 2], for instance, and follow up the bibliographies at the end of these papers. 


\section{\$7. Length functions, ampleness and hyperbolicity}

We have now met several conditions reflecting a certain type of behavior having to do with "positivity":

Ample cotangent bundle;

Hyperbolic;

Ample canonical class;

Pseudo ample canonical class (general type);

Measure hyperbolic.

Previously stated theorems and conjectures already gave some relations between them, notably the last three. Of course we trivially have

$$
\begin{aligned}
\text { Ample cotangent bundle } & \Rightarrow \text { Ample canonical class } \\
& \Rightarrow \text { Pseudo ample canonical class. }
\end{aligned}
$$

None of the reverse implications are true in general. We shall now discuss how to fit hyperbolicity in this chain of implications.

Theorem 7.1 (Kobayashi [Ko 4] Corollary 6.3). Let $X$ be a non-singular variety whose cotangent bundle is ample. Then $X$ is hyperbolic.

Kobayashi's proof is to show that the formalism of Ricci forms and the Schwarz lemma works for something more general than volume forms, pseudo volume forms and hermitian metrics, namely Finsler structures, which are called "Differentialmetrik" in Grauert-Reckziegel [G-R]. I have found that the name "Finsler structure" acts as a psychological deterrent for a very simple notion which generalizes that of a norm, and which has proved useful in practice. In agreement with Griffiths, let us define a length function on a complex vector bundle $E$ over $X$ to be a function into the reals $\geqq 0$,

$$
l: E \rightarrow \mathbf{R}_{\geqq 0}
$$

such that for all complex numbers $c$ and $v \in E$ we have:

LF $1 . l$ is continuous, and $C^{\infty}$ outside the zero section of $E$.

LF 2. $l(v)=0$ if and only if $v=0$.

LF 3. $l$ is absolutely homogeneous of degree 1 , which means

$$
l(c v)=|c| l(v) .
$$

A "Finsler structure" is the square of a length function. It does away with the triangle inequality, and weakens the smoothness conditions of a hermitian metric. It is a hermitian metric if the rank of $E$ is 1 . Instead of writing $l(v)$, we shall also write more suggestively

$$
l(v)=|v|
$$

with the usual absolute value sign.

Let $\pi: \mathbf{P} E \rightarrow X$ be the projective bundle of lines in $E$. Then $\mathbf{P} E$ has the tautological line bundle $L$ on $\pi^{*} E$. As Kobayashi remarks, the length function on $E$ then defines a length function on $L$, which is a smooth hermitian metric because $L$ is one-dimensional. Then Kobayashi defines the curvature and proves for this situation the standard theorems for hermitian metrics. 
For the convenience of the reader, I reproduce an argument of Griffiths giving Kobayashi's Theorem 7.1 directly. So assume that the cotangent bundle $T \vee$ is ample. Then there is an appropriate symmetric power $S^{m} T^{\vee}$ of the cotangent bundle such that a basis of its sections $s_{0}, \ldots, s_{N}$ gives an imbedding of $X$ in the Grassmanian. In fact, if $\mathbf{P T}$ is the projective bundle of lines in the tangent bundle, then we get a projective imbedding

by

$$
\varphi_{m}: \mathbf{P} T \rightarrow \mathbf{P}^{N}
$$

$$
v \mapsto\left(s_{0}\left(v^{m}\right), \ldots, s_{N}\left(v^{m}\right)\right) .
$$

On the hyperplane bundle of $\mathbf{P}^{N}$ we have the tautological (or projective) metric defined in terms of the projective coordinates $w_{0}, \ldots, w_{N}$ by the function

$$
\rho_{i}\left(w_{0}, \ldots, w_{N}\right)=\sum_{j=0}^{N}\left|w_{j} / w_{i}\right|^{2} \text { on the open set } w_{i} \neq 0 \text {. }
$$

Its Chern form is positive and defines the Fubini-Study metric. The inverse image $\varphi_{m}^{*}$ of this tautological metric is a metric $\rho$ on $L$ which defines a length function $l$ on $T$ by

$$
|v|^{2 m}=\sum_{j=0}^{N}\left|s_{j}\left(v^{m}\right)\right|^{2}
$$

We let $c_{1}(\rho)$ be the Chern form of the above metric on $L$, so that $c_{1}(\rho)$ is a positive $(1,1)$-form on $\mathbf{P T}$. Then by compactness, there is a constant $B$ such that

$$
c_{1}(\rho) \geqq B \pi^{*}\left(l^{2}\right) .
$$

A non-constant holomorphic map $f: \mathbf{D} \rightarrow X$ can be lifted to a map $f_{\mathbf{P}}: \mathbf{D} \rightarrow \mathbf{P} T$, making the following diagram commutative.

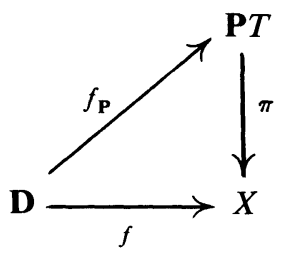

At a point, the lifting is given by $z \mapsto(f(z), d f(z) v)$ wherever $d f(z) \neq 0$, and $v$ is any non-zero complex tangent vector. The extension to the set of isolated singular points is due to the fact that a meromorphic map of an open subset of $\mathbf{C}$ into a projective variety is in fact holomorphic, because at any point, one can factor out a common factor where all projective coordinates may have a common zero or pole. Then $f^{*}\left(l^{2}\right)=f_{\mathbf{P}}^{*} \pi^{*}\left(l^{2}\right)$, so

$$
f_{\mathbf{P}}^{*} c_{1}(\rho) \geqq B f^{*}\left(l^{2}\right) \text {. }
$$

Thus the length function behaves just like a hermitian metric, and its pull-back to $\mathbf{D}$ is a hermitian pseudo metric on $\mathbf{D}$ whose Chern form is positive, and whose Griffiths function is $\geqq B$. The analogues of Theorems 4.2 and 4.3 then work in the present case, and we conclude that $X$ is hyperbolic. 
In general, Kobayashi defines the Ricci form of a length function, and discusses systematically how it relates to the Ricci form on the tautological line bundle of an arbitrary holomorphic bundle. See his Theorems 4.1 and 5.1 of [Ko 4]. These theorems lead into questions of the existence of $(1,1)$-forms or length functions in the direction of a converse for Theorem 4.3. One problem lies in the smoothness of the length function. In Royden [Ro], the length function associated with the Kobayashi distance on a hyperbolic variety is only upper semicontinuous.

Concerning the canonical class itself, as far as I know, the following is an open question.

Conjecture 7.2. If $X$ is non-singular and hyperbolic then the canonical class is ample.

Sommese tells me that hyperbolic together with pseudo canonical does imply ample canonical class in dimenion $\leqq 3$. Conjecture 5.6 would show that the hypothesis of pseudo canonical is redundant. Conversely:

$A$ non-singular hypersurface $X$ of degree $d \geqq n+2$ in $\mathbf{P}^{n}$ has ample canonical class.

Proof. The adjunction formula immediately implies that the canonical sheaf on $X$ is $\mathcal{O}_{X}(-n-1+d)$, and $d \geqq n+2$ is precisely the condition which makes the canonical sheaf ample.

Since the Fermat hypersurfaces

$$
x_{0}^{d}+\cdots+x_{n}^{d}=0
$$

contain lines, we see that in general the condition that $X$ has ample canonical class does not imply $X$ hyperbolic. When the canonical sheaf is not ample, then the hypersurface has a tendency to have lines. Changes of behavior presumably occur for $d=n+1, n, n-1$.

Let $X$ be a hypersurface of degree $d$ in $\mathbf{P}^{n}$. If $d \leqq n-1$ then $X$ contains a line through every point.

This result is classical and easy. Given a point $P$ we can represent it as the origin $P=(0, \ldots, 0)$ on affine $n$-space $\mathbf{A}^{n}$. Let $f=0$ be the affine equation for $X$ on this space. Decompose

$$
f=f_{1}+\cdots+f_{d}
$$

into its homogeneous components of degrees $1, \ldots, d$. Then we get a line through $P$ in $\mathbf{P}^{n}$ if we can solve simultaneously the homogeneous equations

$$
f_{1}=\cdots=f_{d}=0 \text { in } \mathbf{P}^{n-1} .
$$

The condition $d \leqq n-1$ is precisely the inequality which guarantees that this can always be done, for trivial dimensional reasons.

The existence of rational curves in a variety with canonical class which is not numerically effective has long been a subject of interest to algebraic geometers, and has received especially significant impetus in the last few years through the work of Mori, see for instance [Mo]. Such algebraic geometers work over an 
algebraically closed field, and there is of course the diophantine question of whether rational curves are defined over a given field of definition for the variety or hypersurface. One of Mori's theorems states:

If $X$ is a non-singular projective variety, and the tangent bundle is ample, then $X$ is birationally equivalent to projective space (previously Hartshorne's conjecture ).

This is valid over the algebraic closure of a field of definition, and applies to hypersurfaces when $d \leqq n$. Over a given field of definition, the variety may not have any rational points, or may be only unirational, that is, rational image of projective space.

Green [Gr 1] showed that the only non-constant holomorphic maps of $\mathbf{C}$ into a Fermat hypersurface of degree $d$ in $\mathbf{P}^{n}$ are the "Fermat" ones if $d \geqq n^{2}$. For instance, for a given subset of indices, say $0, \ldots, r(r \leqq n-2)$, let $c_{1}, \ldots, c_{r}$ be numbers such that

$$
1+c_{1}^{d}+\cdots+c_{r}^{d}=0
$$

Then $\left(t, c_{1} t, \ldots, c_{r} t\right)$ gives a Fermat line, and all Fermat lines are constructed in this way for each partition of the set of indices. Note that the existence of Fermat lines over a given field, say the rational numbers, amounts to finding rational points on the Fermat hypersurface itself, possibly with fewer variables.

Euler was already concerned with the problem of finding rational curves, that is solving the Fermat equation with polynomials. Swinnerton-Dyer [Sw-D] gives explicit examples of rational curves over the rationals, on

$$
x_{0}^{5}+\cdots+x_{5}^{5}=0 .
$$

Here $X$ has degree $d=n=5$, and thus the canonical class is very non-ample. Swinnerton-Dyer says: "It is very likely that there is a solution in four parameters, or at least that there are an infinity of solutions in three parameters, but I see no prospect of making further progress by the methods of this paper." In general, I conjecture that if $d=n$ then the rational curves are Zariski dense, and even that $X$ is unirational over $\mathbf{Q}$. Actually, when $d=n=3$, the Fermat hypersurface has Ramanujan's taxicab rational point (1729 is the sum of two cubes in two different ways: 9,10 and 12,1). Joe Buhler has verified on the computer that this point satisfies the hypothesis of Theorem 12.11 in Manin's book Cubic Forms, Chapter II, and by that theorem, it follows that in this case, the Fermat hypersurface is indeed unirational over $\mathbf{Q}$. It would be worth while to treat systematically the Fermat hypersurfaces from the present point of view of algebraic geometry, for the existence of rational curves both geometrically and over $\mathbf{Q}$, and for the possibility of their being rational images of projective space for low degrees compared to $n$.

Kobayashi raised the following possibility [Ko 2], p. 71, and Chapter IX, Problem 4:

Let $X$ be a generic complete intersection of hypersurfaces of degrees $d_{1}, \ldots, d_{r}$ in $\mathbf{P}^{n}$. Let $d=d_{1}+\cdots+d_{r}$. If $d \geqq n+2$, then $X$ is hyperbolic. 
If $X$ is a non-singular complete intersection of hypersurfaces of degrees $d_{1}, \ldots, d_{r}$ respectively, then the canonical sheaf on $X$ is

$$
\mathcal{O}_{X}(-n-1+d) \text {, }
$$

where $d=d_{1}+\cdots+d_{r}$ is the sum of the degrees. Hence again the condition for the canonical class to be ample is $d \geqq n+2$. When $d$ is in a range where the Brody-Green example applies, then the hyperbolicity of the generic hypersurface of that degree would follow from Brody's theorem that hyperbolicity is an open condition, if one had an algebraic characterization of hyperbolicity.

\section{§8. Jet differentials}

Interest in classical questions raised by Bloch was revived by Ochiai [Och]. Both introduced jets into the picture. Bogomolov [Bo] then used symmetric differentials. This was followed by the work of Green-Griffiths [Gr-Gr] from the point of view of jet differentials and curvature. The reader will find an extensive bibliography at the end of [Gr-Gr]. I shall now describe jet differentials, and state a conjecture and results of Green-Griffiths which give greater insight into the exceptional locus of pseudo canonical varieties.

Let $X$ be a non-singular variety, and let $x \in X$. Let $\mathbf{D}_{r}$ be a disc as usual, and consider germs of holomorphic mappings $f: \mathbf{D}_{r} \rightarrow X$ such that $f(0)=x$. In local holomorphic coordinates, such an $f$ is given by a convergent power series

$$
f(z)=a_{0}+a_{1} z+a_{2} \frac{z^{2}}{2 !}+\cdots
$$

with $a_{k}=a_{k}(f)=f^{(k)}(0) \in \mathbf{C}^{n}$. Given two such maps $f, g$ we say that they osculate to order $k$ if

$$
f^{(i)}(0)=g^{(i)}(0) \text { for } i=0, \ldots, k .
$$

Equivalence classes of such germs will be called jets of order $k$ at $x$, and the set of such classes will be denoted by $J_{k}(X)_{x}$. We let

$$
J_{k}(X)=\bigcup_{x \in X} J_{k}(X)_{x} \text {. }
$$

Taking obvious charts makes $J_{k}(X)$ into a complex manifold of dimension $n+k n$, and if $U$ is an open set in $X$ with holomorphic coordinates, then this choice of coordinates gives a holomorphic isomorphism

$$
J_{k}(U) \approx U \times \mathbf{C}^{k n} \text {. }
$$

Note that even though the fiber of $J_{k}(X)$ at $x$ is complex analytically isomorphic to $\mathbf{C}^{k n}$, nevertheless $J_{k}(X)$ is not a vector bundle. There is a natural projection

$$
J_{k+1}(X) \rightarrow J_{k}(X)
$$

whose fibers are affine bundles, and whose associated vector bundle is the tangent bundle $T(X)$.

There is a natural action of $\mathbf{C}^{*}$ on $J_{k}(X)$. For $t \in \mathbf{C}^{*}$, in terms of coordinates as above, the action of $t$ is represented by

$$
t\left(a_{0}, a_{1}, \ldots, a_{k}\right)=\left(a_{0}, t a_{1}, t^{2} a_{2}, \ldots, t^{k} a_{k}\right) .
$$


We may call this a weighted action (cf. Dolgachev [Do]). We let

$$
\begin{aligned}
J_{k}^{*}(X)= & \text { non-constant jets, that is those with some } a_{j} \neq 0 \text { for } \\
& 1 \leqq j \leqq k . \\
P_{k}(X)= & J_{k}^{*}(X) / \mathrm{C}^{*} \quad \text { with projection } \pi: P_{k}(X) \rightarrow X .
\end{aligned}
$$

We call $P_{k}(X)$ the weighted projective bundle over $X$. Its fibers may be singular because the action of $\mathbf{C}^{*}$ has fixed points.

We record here that the projection $\pi: P_{k}(X) \rightarrow X$ is proper.

Of course, one can define the weighted projective bundle purely algebraically, but we have put ourselves immediately in the complex analytic context because of the specific applications which follow.

Let $f: \mathbf{D}_{r} \rightarrow X$ be holomorphic. Let $f(z)=x$. Then by taking the Taylor expansion of $f$ up to order $k$ at the given point, in terms of a chart at $x$, we can define a $k$-jet

$$
j_{k}(f)_{z, x}
$$

which is an element of $J_{k}(X)_{x}$. In this way we obtain a map

$$
\mathbf{D}_{r} \rightarrow J_{k}(X) \text {. }
$$

One can put the analogue of length functions on the bundle $P_{k}(X)$, taking the weighted action of $\mathbf{C}^{*}$ into account. Green-Griffiths then consider the analogue of the Chern form

$$
d d^{c} \log \left|j_{k}(f)\right|^{2}
$$

for pseudo length functions on the jet set, and maps $f$ as above. Comparing this form with $\left|j_{k}(f)\right|^{2}$ they extend the formalism of the Ricci form to this jet set. In such applications, they substitute negative curvature arguments (positivity of the analogue of the Griffiths function) for Nevanlinna theory as in Ochiai [Och]. See their comments, e.g. on p. 64.

In this context, Noguchi's theorem (mentioned in §5), and Kobayashi's Theorem 6.1 are jet setting to order 1 .

Just as with ordinary projective space, there is a tautological sheaf $\mathscr{L}_{k}$ over $P_{k}(X)$ which may not be invertible. However, for all $m$ divisible by $k$ ! the tensor power $\mathscr{L}_{k}^{m}$ is invertible, and so can be identified with a line bundle. See [Gr-Gr], p. 46. Because of this, we can define pseudo ampleness for $\mathscr{L}_{k}$ just as we did previously, since the definition involves only large values of $m$ ordered by divisibility.

Conjecture 8.1 (Green-Griffiths). Let $X$ be pseudo canonical. Then for $k$ large, the tautological sheaf $\mathscr{L}_{k}$ is pseudo ample.

We recall that this means that high tensor powers $L_{k}^{m}$ have maximal Kodaira dimension, or equivalently, the rational map into projective space

$$
\varphi_{m}=\left(s_{0}, \ldots, s_{N}\right): P_{k}(X) \rightarrow \mathbf{P}^{N}
$$

induced by a basis of $H^{0}\left(P_{k}(X), \mathscr{L}_{k}^{m}\right)$ is birational for $m$ sufficiently large ordered by divisibility. The conjecture is proved in [Gr-Gr], Proposition 1.11, 
in the case of surfaces. Also Proposition 1.10 loc. cit. shows that the Euler characteristic of the higher-order jets comes out to be the "right" number in any dimension, thus giving evidence for the higher-dimensional conjecture.

We now suppose that $k$ is sufficiently large. The birational map as above for $m$ sufficiently large has a Bad locus, or base locus, consisting of points of indeterminacy, and the union of positive dimensional fibers of $\varphi_{m}$. Let $B_{k, m}$ be this base locus. Then $B_{k, m}$ stabilizes for $m$ large (ordered by divisibility), so we write it $B_{k}$. Finally we let

$$
S=\bigcap \pi\left(B_{k}\right)
$$

be the Green-Griffiths set.

Theorem 8.2. A non-constant holomorphic map $\mathbf{C} \rightarrow X$ is contained in the Green-Griffiths set.

This theorem holds whether the Green-Griffiths set is a proper subset or not, and is proved in [Gr-Gr], Corollary 2.8. (There is a misprint, the union sign should be an intersection.)

In particular, the exceptional set of Conjecture 5.8 is contained in the Green-Griffiths set $S$. I asked Green-Griffiths whether they might be equal. Green told me that the two sets are not equal in general. Certain Hilbert modular surfaces constructed by Shavel [Sh], compact quotients of the product of the upper half-plane with itself, provide a counterexample which is hyperbolic, but such that the Green-Griffiths set is the whole variety. Thus the jet construction appears insufficient so far to characterize the exceptional set of Conjecture 5.8 completely algebraically. It becomes a problem to find conditions under which they are equal.

I also asked whether the complement $X-S$ is hyperbolic, and unlike the situation in Conjecture 5.8, Green told me he could prove it in this case.

\section{Historical appendix: algebraic families}

The various conditions which we have met, influencing the diophantine behavior of a variety, arose in the context of algebraic families. These played no role in the main part of this article, but played an important historical role, as follows.

Instead of a single variety defined over a field $F_{0}$, we consider an algebraic family of varieties $\left\{X_{w}\right\}$, depending on parameters $w$ lying in some parameter variety $W$. Instead of rational points, we consider rational sections of $W$ in the family. This point of view was taken systematically in [La 0]. One can then translate systematically the conjectures about the finiteness of rational points into conjectures about the finiteness of the number of sections. For instance, if the generic fiber $X_{w}$ is hyperbolic and there exist infinitely many sections, then their Zariski closure must split birationally into a product $X_{0} \times W$. In that case, a section comes from a rational map

$$
W \rightarrow X_{0} .
$$


If, for instance, $X_{0}=C \times C$, where $C$ is a curve of genus $\geqq 2$, and $W=C$, then there is a family of rational maps $W \rightarrow X_{0}$ such that $C \rightarrow C \times\{a\}$, with $a \in C$. But in any case, conjecturally, there is only a finite number of surjective rational maps of $W$ onto $X_{0}$, and I told this conjecture to Kobayashi. When $X_{0}$ has dimension 1 , and genus $\geqq 2$, this is a classical theorem of de Franchis. Kobayashi and Ochiai proved the generalization to higher dimension, for the split case of algebraic families, not under the original hypothesis that the generic fiber is hyperbolic, but under the condition that $X_{0}$ is "of general type" [K-O 2]. They conjectured that hyperbolic implies general type. This immediately made me conjecture that for a variety of general type, the set of rational points is not Zariski dense (unpublished). Noguchi [No 1] proved the general conjecture for non-split families under still a third possible condition, that the cotangent bundle is ample. Noguchi also reports that Bombieri made the above conjecture in lectures at Chicago in 1980. For more in this direction, see also [No 2].

In the case of an algebraic family $\left\{X_{w}\right\}$ with generic fiber of dimension 1 -that is, a family of curves - all these conditions coincide, and are equivalent to the condition that the curve has genus $\geqq 2$. The finiteness of the number of sections if the family does not split was originally due to Manin [Ma]. Grauert [Gra] subsequently gave another proof for Manin's theorem, and Noguchi's method extends Grauert's. In this way, the deformation theory of algebraic varieties over the complex numbers influenced the diophantine theory of varieties over finitely generated fields over the rational numbers.

Riebesehl [Ri] took off from Grauert-Reckziegel [G-R], and gave a version of the diophantine result for algebraic families in the higher-dimensional case under a hypothesis of negative curvature (which he takes as his definition of hyperbolicity). He actually uses length functions, and handles the singular case, but he assumes that all the fibers have negative curvature, which is similar to "good reduction" everywhere. This is quite a restrictive assumption.

Despite this restriction, the method of proof is interesting for its own sake, and we sketch it here. Let $\pi: X \rightarrow W$ be the family. In various degrees of generality, Grauert-Reckziegel and Riebesehl prove the following fundamental fact. Let $w$ be a point of the base. Then there exists a neighborhood $V$ of $w$ such that $\pi^{-1}(V)$ has a hyperbolic length function. It then follows from the Ahlfors-Schwarz lemma (the length decreasing property) that the family of sections is a normal family. From this one deduces at once that when $W, X$ are imbedded in projective space, the projective degrees of the sections are bounded. This is the essential part of the diophantine result: sections are of bounded height.

There remains the question of splitting the family. One can then use the techniques from early days [La 0]. The boundedness of the degrees implies that the sections lie in only a finite number of Chow families. If one member of an irreducible component is a section, so is the generic member. Assuming that the family of sections is Zariski dense, it follows that there exists a variety $T$ and a generically surjective rational map

$$
f: T \times W \rightarrow X .
$$


From this one would like to split $X$ birationally, but Riebesehl splits only a curve, using results from [La 0]. More generally, it remains a problem to prove the following, which we state in a self-contained way, under weaker assumptions.

Let $\pi: X \rightarrow W$ be a generically surjective rational map, whose generic fiber is geometrically irreducible and pseudo canonical. Assume that there exists a variety $T$ and a generically surjective rational map

$$
f: T \times W \rightarrow X .
$$

Then $X$ is birationally equivalent to a product $X_{0} \times W$.

The study of algebraic families from the diophantine point of view thus put forth several conditions like hyperbolic, general type, ample cotangent bundle, negative curvature.

Manin's original proof introduced a different aspect of differential geometry into the question, what became known as the Gauss-Manin connection: differentiating with respect to the parameters of the family.

For convenience of language, it was useful to start with a variety defined over the complex numbers. In number theory, one usually starts with a variety defined over a field $F_{0}$ which is finite over the rationals, that is a number field. There was no need to do so here. However, a few remarks on the relations between number fields and arbitrary finitely generated fields may be useful to the reader. A priori, the statement:

(a) A variety of a certain type has only a finite number of rational points in every finitely generated field over $\mathbf{Q}$.

is stronger than

(b) A variety of a certain type, defined by equations over a number field, has only a finite number of rational points in every number field.

However, for varieties of dimension 1 (curves), the two statements are equivalent in the case of curves of genus $\geqq 2$. This can be proved easily by a specialization argument based on a theorem of Néron, using Hilbert's Irreducibility Theorem, see [La 2], Chapter IX, Theorem 6.2, and Chapter XII, Theorem 2.3, including an extension by Silverman. It would be interesting to study the higher-dimensional case from this point of view, which lies somewhere between algebraic families over the complex numbers, and single varieties defined over a number field.

\section{BiBLIOGRAPHY}

[Ax] J. AX, Some topics in differential algebraic geometry II: On the zeros of theta functions, Amer. J. Math. 94 (1972), 1205-1213.

[B1] A. BLOCH, Sur les systèmes de fonctions uniformes satisfaisant à l'équation d'une variété algébrique dont l'irrégularité dépasse la dimension, J. de Math. V (1926), 19-66. 
[Bog] F. BOGOMOLOV, Families of curves on a surface of general type, Soviet Math. Dokl. 18 (1977), 1294-1297.

[Bom] E. BOMBIERI, Canonical models of surfaces of general type, Inst. Hautes Études Sci. Publ. Math 42 (1973), 171-219.

[Br 1] R. BRODY, Intrinsic metrics and measures on compact complex manifolds, thesis, Harvard, 1975.

[Br 2] R. BRODY, Compact manifolds and hyperbolicity, Trans. Amer. Math. Soc. 235 (1978), 213-219.

[Br-Gr] R. BRODY and M. GREEN, $A$ family of smooth hyperbolic hypersurfaces in $P_{3}$, Duke Math. J. 44 (1977), 873-874.

[Ch] S. S. CHERN, On holomorphic mappings of Hermitian manifolds of the same dimension, Proc. Sympos. Pure Math., vol. 11, Amer. Math. Soc., Providence, R. I., 1968, pp. 157-170.

[Di 1] A. DINGHAS, Ein n-dimensionales Analogon des Schwarz-Pickschen Flächensatzes fur holomorphe Abbildungen der komplexen Einheitskugel in eine Kähler-Manningfaltigkeit, Festschrift zur Gedachtnisfeier für Karl Weierstrass, Westdeutscher Verlag, Cologne (1966), 477-494.

[Di 2] A. DINGHAS, Über das Schwarzsche Lemma und verwandte Sätze, Israel J. Math. 5 (1967), 157-169.

[Do] I. DOLGACHEV, Weighted projective varieties, Group Actions and Vector Fields (Proc. Polish-North Amer. Sem., Vancouver, 1981; J. B. Carrels, editor), Lecture Notes in Math., vol. 956, Springer-Verlag, Berlin and New York, 1982, pp. 34-71.

[E] D. A. EISENMAN, Intrinsic measures on complex manifolds and holomorphic mappings, Mem. Amer. Math. Soc. No. 96 (1970).

[Gr-Wu] I. GRAHAM and H. WU, Some remarks on the intrinsic measures of Eisenman, Trans. Amer. Math. Soc. 288 (1985), 625-660.

[Gra] H. GRAUERT, Mordell's Vermutung über rationale Punkte auf algebraische Kurven und Funktionenkörper, Inst. Hautes Études Sci. Publ. Math. 25 (1965), 131-149.

[G-P] H. GRAUERT and U. PETERNELL, Hyperbolicity of the complement of plane curves, Manuscripta Math. 50 (1985), 429-441.

[G-R] H. GRAUERT and H. RECKZIEGEL, Hermitesche Metriken und normale Familien holomorpher Abbildungen, Math. Z. 89 (1965), 108-112.

[Gr 1] M. GREEN, Some Picard theorems for holomorphic maps to algebraic varieties, Amer. J. Math. 97 (1975), 43-75.

[Gr 2] M. GREEN, Holomorphic maps to complex tori, Amer. J. Math. 100 (1978), 615-620.

[Gr-Gr] M. GREEN and P. GRIFFITHS, Two applications of algebraic geometry to entire holomorphic mappings, The Chern Symposium 1979, (Proc. Internat. Sympos., Berkeley, Calif., 1979), Springer-Verlag, New York, 1980, pp. 41-74.

[Gri] P. GRIFFITHS, Holomorphic mapping into canonical algebraic varieties, Ann. of Math. (2) 98 (1971), 439-458.

[Ha-M] J. HARRIS and D. MUMFORD, On the Kodaira dimension of the moduli space of curves, Invent. Math. 67 (1982), 23-86.

[Ha] R. HARTSHORNE, Ample vector bundles, Inst. Hautes Études Sci. Publ. Math., 29 (1966), 319-350.

[K-K] P. KIERNAN and S. KOBAYASHI, Holomorphic mappings into projective space with lacunary hyperplanes, Nagoya Math J. 50 (1973), 199-216.

[Ka 1] Y. KAWAMATA, The cone of curves of algebraic varieties, Ann. of Math. (2) 119 (1984), 603-633. 
[Ka 2] Y. KAWAMATA, Pluricanonical systems on minimal algebraic varieties, Invent. Math. 79 (1985), 567-588.

[Ko 1] S. KOBAYASHI, Volume elements, holomorphic mappings and Schwarz lemma, Proc. Sympos. Pure Math., vol. 11, Amer. Math. Soc., Providence, R. I., 1968, pp. 253-260.

[Ko 2] S. KOBAYASHI, Hyperbolic manifolds and holomorphic mappings, Marcel Dekker, New York, 1970.

[Ko 3] S. KOBAYASHI, Intrinsic distances, measures and geometric function theory, Bull. Amer. Math. Soc. 82 (1976), 357-416.

[Ko 4] S. KOBAYASHI, Negative vector bundles and complex Finsler structures, Nagoya Math. J. 57 (1975), 153-166.

[K-O 1] S. KOBAYASHI and T. OCHIAI, Mappings into compact complex manifolds with negative first Chern class, J. Math. Soc. Japan 23 (1971), 137-148.

[K-O 2] S. KOBAYASHI and T. OCHIAI, Meromorphic mappings into compact complex spaces of general type, Invent. Math. 31 (1975), 7-16.

[La 0] S. LANG, Integral points on curves, Inst. Hautes Études Sci. Publ. Math. 6 (1960), $27-43$.

[La 1] S. LANG, Higher dimensional diophantine problems, Bull. Amer. Math. Soc. 80 (1974), 779-787.

[La 2] S. LANG, Fundamentals of diophantine geometry, Springer-Verlag, 1983.

[La 3] S. LANG, Introduction to transcendental numbers, Addison-Wesley, 1966.

[Ma] J. MANIN, Rational points of algebraic curves over function fields, Izv. Akad. Nauk 27 (1963), 1395-1440.

[Mo] S. MORI, Threefolds whose canonical bundles are not numerically effective, Ann. of Math. (2) 116 (1982), 133-176.

[Mo-Mu] S. MORI and S. MUKAI, The uniruledness of the moduli space of curves of genus 11, Algebraic Geometry Conference, Tokyo-Kyoto, 1982, Lecture Notes in Math., vol. 1016, pp. 334-353.

[Mu] D. MUMFORD, The canonical ring of an algebraic surface, Ann. of Math. (2) 76 (1962), 612-615.

[No 1] J. NOGUCHI, A higher-dimensional analogue of Mordell's conjecture over function fields, Math. Ann. 258 (1981), 207-212.

[No 2] J. NOGUCHI, Logarithmic jet spaces and extensions of de Franchis' theorem (to appear).

[Och] T. OCHIAI, On holomorphic curves in algebraic varieties with ample irregularity, Invent. Math. 43 (1977), 83-96.

[Re] H. RECKZIEGEL, Hyperbolische Raüme und normale Familien holomorpher Abbildungen, Dissertation, Göttingen, 1967.

[Ri] D. RIEBESEHL, Hyperbolische Komplex Raüme und die Vermutung von Mordell, Math. Ann. 257 (1981), 99-110.

[Ro] H. ROYDEN, Remarks on the Kobayashi metric, Several Complex Variables. II (Proc. Internat. Conf. Univ. Maryland, College Park, Md., 1970), Lecture Notes in Math., vol. 185, Springer-Verlag, 1971, pp. 125-137.

[Sh] I. SHAVEL, A class of algebraic surfaces of general type constructed from quaternion algebras, Pacific J. Math. 76 (1978), 221-245.

[Si] C. L. SIEGEL, Über einige Anwendungen diophantischer Approximationen, Abh. Preuss. Akad. Wiss. Phys. Math. Kl. (1929), 41-69. 
[Sw-D] P. SWINNERTON-DYER, $A$ solution of $A^{5}+B^{5}+C^{5}=D^{5}+E^{5}+F^{5}$, Proc. Cambridge Philos. Soc. 48 (1952), 516-518. (Note: Under the name of P. S. Dyer, see also the case of degree 4 in J. London Math. Soc. 1943, extending the work of Euler.)

[Ta] Y.-S. TAI, On the Kodaira dimension of the moduli space of abelian varieties, Invent. Math. 68 (1982), 425-439.

[Vo] P. VOJTA, Springer Lecture Notes, to appear; see also his thesis, Harvard, 1984.

[Wo] J. WOLFART, Taylorentwicklungen automorpher Formen und ein Transzendenzproblem aus der Uniformisierungs theorie, Abh. Math. Sem. Univ. Hamburg 54 (1984), $25-33$.

[W-W] J. WOLFART and G. WUSTHOLZ, Der Überlagerungsradius algebraischer Kurven und die Werte der Betafunktion an rationalen Stellen (to appear).

[Ya] S. T. YAU, Intrinsic measures on compact complex manifolds, Math. Ann. 212 (1975), 317-329.

Department of Mathematics, Yale University, New Haven, Connecticut 06520 
